\title{
Verification of Safety Properties for Parameterized Regular Systems
}

\author{
DAVID CACHERA and KATELL MORIN-ALLORY \\ IRISA
}

\begin{abstract}
We propose a combination of heuristic methods to prove properties of control signals for regular systems defined by means of affine recurrence equations (AREs). We benefit from the intrinsic regularity of the underlying polyhedral model to handle parameterized systems in a symbolic way. Our techniques apply to safety properties. The general proof process consists in an iteration that alternates two heuristics. We are able to identify the cases when this iteration will stop in a finite number of steps. These techniques have been implemented in a high level synthesis environment based on the polyhedral model.

Categories and Subject Descriptors: B.6.3 [Logic Design]: Design Aids; D.2.4 [Software Engineering]: Software/Program Verification; F.3.1 [Logics and Meanings of Programs]: Specifying and Verifying and Reasoning about Program

General Terms: Verification, Design

Additional Key Words and Phrases: Polyhedral model, parameterized systems, regular systems, embedded systems, codesign
\end{abstract}

\section{INTRODUCTION}

The increasing complexity of embedded systems calls for the elaboration of mechanical, modular and secure codesign tools. The design of a complex and heterogeneous system can no longer rely on a hand-performed composition of software and hardware components whose interfaces are informally specified.

The combination of recurrence equations [Karp et al. 1967] over polyhedral domains and affine dependency functions is the basis of the so-called polyhedral model. This model provides a unified framework for expressing hardware and software parts of regular systems. Systems are described in a generic manner through the use of symbolic parameters, and structuring mechanisms allow for hierarchical specifications. The ALPHA language [Wilde 1994] and the MMALPHA environment [Wilde 1993] provide a syntax and a programming environment to define and manipulate polyhedral equational systems.

Authors' address: IRISA, Campus de Beaulieu F-35042 Rennes, France

Permission to make digital or hard copies of part or all of this work for personal or classroom use is granted without fee provided that copies are not made or distributed for profit or direct commercial advantage and that copies show this notice on the first page or initial screen of a display along with the full citation. Copyrights for components of this work owned by others than ACM must be honored. Abstracting with credit is permitted. To copy otherwise, to republish, to post on servers, to redistribute to lists, or to use any component of this work in other works requires prior specific permission and/or a fee. Permissions may be requested from Publications Dept., ACM, Inc., 1515 Broadway, New York, NY 10036 USA, fax: +1 (212) 869-0481, or permissions@acm.org. (C) 2005 ACM 1539-9087/05/0500-0228 $\$ 5.00$ 
Systems are first expressed in a high-level manner, close to the initial algorithmic specification. Then they are refined through a user-guided series of automatic transformations, down to an implementable description, specifying in detail how, where and when each computation should take place. From this low-level description, we may derive either $\mathrm{C}$ code (under the form of nested loops) or an architectural description in VHDL. When the system is partitioned into hardware and software components, interfaces are generated to control communication between both parts [Derrien et al. 2002]. For hardware components and interfaces, control signals are generated to validate computations or data transfers.

The use of systematic and semi-automatic rewritings together with the clean semantic basis provided by the polyhedral model should ensure the correctness of the final implementation. Nevertheless, the development of real-size systems results in a loss of confidence in the initial high-level specification. Moreover, interface and control signal generators are not certified, and hand-made optimizations are still performed to tune the final result. As a consequence, even if functional correctness is guaranted by the refinement process from an initial algorithmic specification, the corectness of control signals has to be checked at a lower level of description. This calls for the development of a formal verification tool to (partially) certify low-level system descriptions before their final implementation.

In this paper, properties about control signals, that is, boolean signals used to control the computation flow, will be referred to as control properties. Of course, the techniques developed here apply to any kind of boolean signal in our model. Our aim is thus to develop proof methods for control properties. We will take advantage of the regularity of the polyhedral model, that is, its ability to model regular systems size of which depends on symbolic parameters. This model naturally provides a finite representation for systems size of which is not a priori known.

Polyhedra have been widely used to represent sets of integer values in hybrid automata, thus providing abstraction for a restricted class of infinite state systems (see Henzinger et al. [2001], Silva and Krogh [2000], Asarin et al. [2002], Alur et al. [2000] for instance). In contrast, our approach does not use polyhedra to model the variable values, but the index space of variable instances. Existing tools for manipulating polyhedra will allow us to "hide" inductive proofs behind syntactic substitutions, while the analysis of affine or uniform dependencies will help us in automatically finding invariants. This combination of polyhedra manipulations and automatic invariant searching has been implemented in the MMALPHA environment and proves successful for most properties of parameterized systems described at bit level.

The rest of this paper is organized as follows. In Section 2, we show on two very simple examples the kind of properties we want to prove. In Section 3, we briefly present the polyhedral model. In Section 4, we sketch on an example the whole proof process. Section 5 is devoted to a substitution method that allows one to perform inductive proofs without explicitly expressing the induction step. In Section 6, we show how to look for invariants in more complex cases, while Section 7 explains how and when we combine these two methods. Section 8 is 


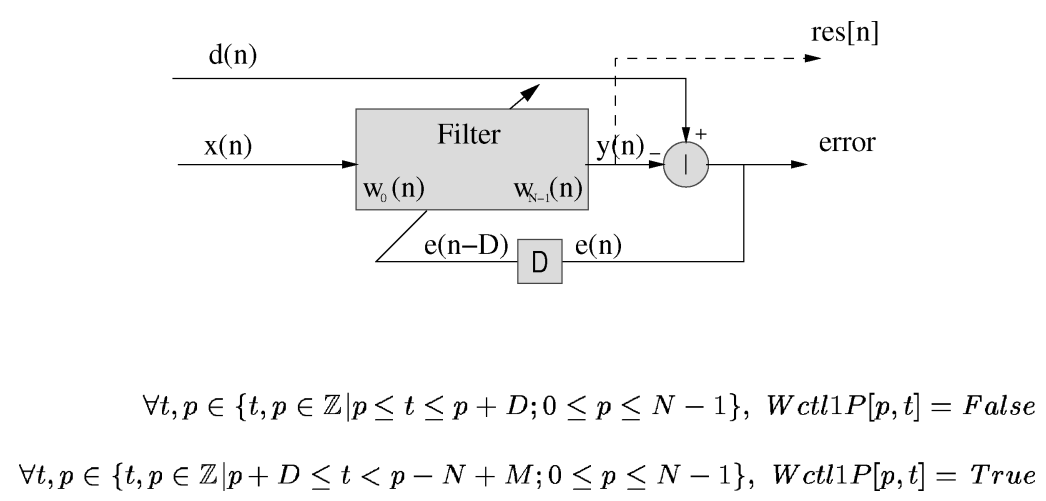

Fig. 1. An adaptive filter: General stucture and properties to prove.

devoted to the implementation of the proof process. In Section 9, we come back to our examples to show how our proof method applies. Section 10 gives a brief insight into the overall complexity of our method. Section 11 gives an overview of related work, before we give concluding remarks in Section 12.

\section{MOTIVATING EXAMPLES}

To motivate our approach, let us introduce two simple concrete examples. The first example deals with an adaptive filter delayed least mean square (DLMS) [Guillou et al. 2000]). The system (cf. Figure 1) consists of $N$ cells, each one storing a weight in a register. These cells are organized in a linear array. There are two inputs $x$ and $d$, respectively for the raw input signal and for the desired output, and one output res. The system computes a convolution between input $x$ and weights $w[i]$, then computes an error signal by subtracting the result by the desired output. This error is propagated backwards to update weights with a delay $D$. Under certain statistical assumptions on inputs, weights are converging to the desired values. This system is parameterized by parameters $N, M, D: N$ is the filter length (number of weights), $D$ the delay and $M$ the length of input flow $x$. In our example, due to the error feedback, some registers are not accessible as long as the output is not defined. The internally generated signal $W c t l 1 P$ is used to control the access to registers $w[i]$. This signal is defined on set $\{p, t \in \mathbb{Z} \mid p \leq t \leq p-N+M ; 0 \leq p \leq N-1\}$, where parameters $N, M, D$ are defined on set $\{N, M, D \in \mathbb{Z} \mid 3 \leq N \leq \min (M-D-1, D-1)\}$. As long as the system is in its initialization phase, due to the feedback delay, weights are not correctly defined, so Wctl1P must be false in order to prevent access to these weights. In a second phase, this signal must become and stay true to allow access to the registers containing weights. Initial values for this signal are generated in the first unit, and then pipelined in the entire array. We want to prove that for any value of the symbolic parameters $N, M, D$, signal $W c t l 1 P$ is false for all indices in the set $\{t, p \in \mathbb{Z} \mid p \leq t \leq p+D ; 0 \leq p \leq N-1\}$, and that this same signal is true for all indices in the set $\{t, p \in \mathbb{Z} \mid p+D \leq t<$ $p-N+M ; 0 \leq p \leq N-1\}$.

The second example is a system computing a matrix-vector product (cf. Figure 2). The system consists of a linear array of $N$ cells, separated one from 


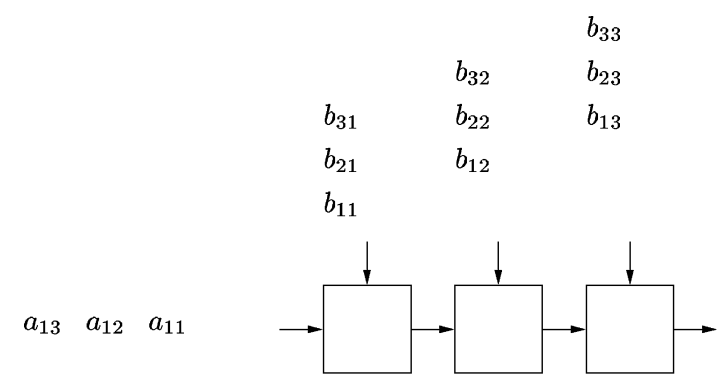

(a) The array and its data inputs, for $N=3$

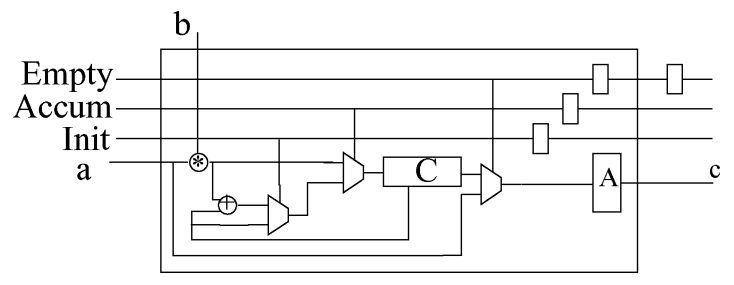

(b) One cell

$\forall t \in\{t \in \mathbb{Z} \mid N<t\}, c[t]=$ True

(c) Property to prove

Fig. 2. Structure of an array computing a matrix-vector product.

another by a register. Each cell has two data inputs ( $a$ and $b)$, three control inputs (Init, Empty, and Accum), and one data output (c). Inputs $a$ are vector coefficients, and inputs $b$ are matrix coefficients. Each cell contains one register $C$ in which the local result of each computation is stored. Control signal Init is used to initialize register $C$, signal Accum to accumulate the product of coefficients in $C$ and signal Empty to output it in register $A$. Register $A$ is either the result of a coefficient computation, or a vector coefficient. Our problem does not lie in knowing precisely what the result is, but in ensuring that it always has a significant value. More precisely, inputs enter the array with the following scheme: in each cell $N$ matrix coefficient values are input ( $N$ being the matrix size), followed by $N$ "empty" values, until the whole matrix has been handled. On the output, the initial $N$ vector coefficients first get out, followed by $N$ computations of coefficients of the result vector. On this particular example, we want to prove that the output is always significant.

Since we are just interested in the presence or absence of a significant result, we will work on a simpler version of the system, by modeling integer variables by boolean variables. An instance of a variable is true if the corresponding integer instance is significant, otherwise it is false. Furthermore, we model operations on integer variables by the conjunction of the corresponding boolean operands: the result of an integer operation is significant if and only if all the operands are significant.

Here, output $c$ cannot be true before the first coefficient is used in the last cell. The precise property we prove is the following: for all $t$ in $\{t \in \mathbb{Z} \mid N<t\}$, $c[t]$ is true. 
In both examples, we are not interested in proving a full functional specification of the system, but rather in automatically checking a simple property concerning control signals that might have been introduced by hand.

In the following, we will work on multidimensional variables. As we have seen of the examples, these variables are defined on polyhedral domains, i.e., sets of index vectors of polyhedral shape in $\mathbb{Z}^{n}$. We will only deal with safety properties, i.e., we will always consider the problem of proving that a signal is true (or false) on a given polyhedral domain.

\section{THE POLYHEDRAL MODEL}

We now present the basic concepts underlying the polyhedral model. We will define the notion of System of Affine Recurrence Equations (SARE), then we will introduce the notions of schedule, of syntactic transformation of a SARE, and give a brief insight into their semantics. In the following, we will denote by $\mathbb{N}, \mathbb{Z}, \mathbb{Q}$, and $\mathbb{R}$ the sets of, respectively, natural numbers, integers, rational, and real numbers.

\subsection{Polyhedral Domains and Recurrence Equations}

Definition 3.1 (Polyhedral Domain). An $n$-dimensional polyhedron is a subset $\mathcal{P}$ of $\mathbb{Q}^{n}$ bounded by a finite number of hyperplanes. It can be implicitly defined by: $\mathcal{P}=\left\{x \in \mathbb{Q}^{n} \mid A . x \leq b\right\}$ where $A \in \mathbb{Q}^{m \times n}$ and $b \in \mathbb{Q}^{m}$.

We call polyhedral domain (or, for short, domain) a subset $\mathcal{D}$ of $\mathbb{Z}^{n}$ defined by

$$
\mathcal{D}=\mathbb{Z}^{n} \cap \mathcal{P}
$$

where $\mathcal{P}$ is a finite union of $n$-dimensional polyhedra.

Definition 3.2 (Variable). A variable $X$ is an application from an $n$ dimensional domain into a base set (booleans, integers, or reals); it is said to be an $n$-dimensional variable. We call instance of the variable $X$ any restriction of $X$ to a single point $z$ of its domain, and denote it by $X[z]$. Constants are associated to the trivial domain $\mathbb{Z}^{0}$.

Definition 3.3 (Recurrence Equation). A recurrence equation defining a function (variable) $X$ at all points, $z$, in a domain, $\mathcal{D}_{X}$, is an equation of the form

$$
X=\mathcal{D}_{X}: g(\ldots, X . d, \ldots)
$$

where

$-X$ is an $n$-dimensional variable;

$-d$ is a dependency function, $d: \mathbb{Z}^{n} \rightarrow \mathbb{Z}^{n}$;

$-g$ is a strict function;

$-\mathcal{D}_{X}$ is a set of points in $\mathbb{Z}^{n}$ and is called the domain of the equation. 
A variable may be defined by more than one equation. In this case, we use the syntax shown below,

$$
X=\left\{\begin{array}{c}
\vdots \\
\mathcal{D}_{X_{i}}: g_{i}\left(\ldots X . d_{i} \ldots\right) \\
\vdots
\end{array}\right.
$$

Each line is called a branch, and the domain of $X$ is the union of the (disjoint) domains of all the branches, $\mathcal{D}_{X}=\bigcup_{i} \mathcal{D}_{X_{i}}$. We also say that the dependency function $d_{i}$ holds over the (sub) domain $D_{X_{i}}$.

Definition 3.4 (Affine Recurrence Equation). A recurrence equation as defined above, is called an affine recurrence equation (ARE) if every dependency function is of the form, $d(z)=A z+a$, where $A$ is an $n \times n$ matrix and $a$ is an integer $n$-vector. If matrix $A$ is the identity matrix, dependency $d$ is said to be uniform. If all the dependencies are uniform, the equation is said to be a uniform recurrence equation (URE).

Definition 3.5 (System). A system of affine recurrence equations (SARE) is a set of $m$ equations like (1), defining the data variables $X_{1} \ldots X_{m}$. Each variable $X_{i}$ is of dimension $n_{i}$, and since the equations may now be mutually recursive, the dependency functions $d$ must now have the appropriate dimensionality.

\subsection{Dependency Graphs and Schedules}

In order to get a valid implementation from a set of recurrence equations, we need (among other things) to determine a particular order in which computations should take place. The definitions above do not specify such an order, and do not even guarantee that it may exist. Recurrence equations define dependencies between elementary computations, thus constraining the set of possible valid orders.

Definition 3.6 (Dependency Graph). We say that an instance $X[z]$ of a variable $X$ depends on an instance $Y\left[z^{\prime}\right]$ of a variable $Y$ if there exists an equation of the form $X=g(\ldots, Y . d, \ldots)$ where $z^{\prime}=d(z)$. We denote by $X[z] \rightarrow Y\left[z^{\prime}\right]$ this fact.

We call the dependency graph the graph the vertices of which are the variable instances, and where there is an edge between vertices $X[z]$ and $Y\left[z^{\prime}\right]$ if and only if $X[z] \rightarrow Y\left[z^{\prime}\right]$.

We call the reduced dependency graph the graph the vertices of which are the multi-dimensional variables; there is an edge from variable $X$ to variable $Y$ (denoted by $X \rightarrow Y$ ) iff there exists an instance $X[z]$ of $X$ and an instance $Y\left[z^{\prime}\right]$ of $Y$ such that $X[z] \rightarrow Y\left[z^{\prime}\right]$.

Definition 3.7 (Schedule). A schedule $t_{X}$ is a function such that $t_{X}(z)$ specifies the time instant at which $X[z]$ is computed. Typically, the range of $t_{X}$ is $\mathbb{N}$, but any total order is allowed (for example $\mathbb{N}^{k}$ under the lexicographic order). In the following, we restrict ourselves to one-dimensional schedules. A schedule 
is said to be valid ${ }^{1}$ if for any instances $X[z]$ and $Y\left[z^{\prime}\right]$,

$$
X[z] \rightarrow Y\left[z^{\prime}\right] \Longrightarrow t_{X}(z)>t_{Y}\left(z^{\prime}\right) .
$$

A set of recurrence equations is said to be schedulable if such a valid schedule exists. The problem of determining if a SARE is schedulable is undecidable in the general case (see Saouter and Quinton [1993]), but much work has been devoted to the development of heuristics to find schedules respecting particular formats.

\subsection{System Transformations}

The combination of polyhedral domains and affine dependency functions is the basis of the so-called polyhedral model. Closure properties in this model (namely by intersection, finite union and inverse of an affine application) are the key of a rich set of formal correctness preserving transformations. The most important manipulation that we can perform on a SARE is called a reindexing transformation (also called a change of basis or space-time mapping) to its variables. This transformation must admit a left inverse for all points in the domain of the variable. Let a variable $X_{p}$ of dimension $d$ of a SARE be defined as follows:

$$
X_{p}=\left\{\begin{array}{c}
\vdots \\
\mathcal{D}_{X_{p, i}}: g_{i}\left(\ldots X_{q} \cdot d_{i} \ldots\right) \\
\vdots
\end{array}\right.
$$

Let $\mathcal{T}$ be a mapping from $\mathbb{Z}^{d}$ into $\mathbb{Z}^{d}$. Applying reindexing transformation $\mathcal{T}$ to $X_{p}$ consists in the following steps ${ }^{2}$ :

-Replace each $\mathcal{D}_{X_{p, i}}$ by $\mathcal{T}\left(\mathcal{D}_{X_{p, i}}\right)$.

- On the rhs of the equation for $X_{p}$, replace each dependency $d_{i}$ by $d_{i} \circ \mathcal{T}^{-1}$, the composition of $d_{i}$ and $\mathcal{T}^{-1}$.

-In all occurrences $X_{p} \cdot g$ on the rhs of any equation, replace the dependency $g$ by $\mathcal{T} \circ g$.

The SARE we obtain after this transformation is semantically equivalent to the original.

From now on, we only consider schedulable systems, which are the only systems usable in practice. Moreover, we consider that all systems have been scheduled: for each variable $X$,

- a valid schedule $t_{X}$ has been determined, and

- a reindexing transformation based on $t_{X}$ has been applied to $X$. After this transformation, the first index of the domain of $X$ is considered as the

\footnotetext{
${ }^{1}$ This definition is a bit restrictive but sufficient for our purpose. We can relax the constraint by requiring only $t_{X}(z) \geq t_{Y}\left(z^{\prime}\right)$. In that case, we have to verify additional constraints on the dependency graph to ensure the validity of the schedule.

${ }^{2}$ Recall that, since equations are mutually recursive, $X_{p}$ depends on some other variables $X_{q}$.

ACM Transactions on Embedded Computing Systems, Vol. 4, No. 2, May 2005.
} 
temporal index. Unless otherwise specified, this particular index will be denoted by $t$.

\subsection{Semantics}

We shall now give a brief insight into the semantics of SAREs. We use the denotational semantics defined in [Mauras 1989] (see this reference for details). As we focus on control properties, we only deal with boolean variables and expressions. The base semantic domain will thus be the following lattice:

$$
\mathbb{B}=\{t t, f f, \perp, \top\}
$$

where these elements respectively represent true, false, the undefined value (minimum of the lattice), and the erroneous value (maximum of the lattice).

A multidimensional value is a mapping from an index space to the base scalar values,

$$
V A L=\mathbb{Z}^{n} \rightarrow \mathbb{B} .
$$

An environment $\rho$ maps a variable to a multidimensional value,

$$
\rho \in E V=V A R \rightarrow V A L .
$$

The semantics $\mathcal{E}(e)$ of an expression $e$ maps an environment to a multidimensional value,

$$
\mathcal{E}(e) \in E X P=E V \rightarrow V A L .
$$

It is defined by induction on the expression's structure.

The semantics of an equation maps an environment to an environment and is defined by

$$
\mathcal{Q}(X=e)(\rho)=\rho(\sup (\rho(X), \mathcal{E}(e)(\rho)) / X)
$$

where sup is the least upper bound in $\mathbb{B}$, and $\rho(V / X)$ denotes the substitution of $X$ by the (multidimensional) value $V$ in $\rho$. Finally, the semantics of a system of equations is the least fixpoint of the composition of the semantics of all equations.

An environment that is correct w.r.t. the semantics of a given SARE will be called semantically correct. More formally, we give the following definitions.

Definition 3.8 (Validity). Let $\rho$ be an environment, $e$ an expression, $z$ an index in the definition domain $\mathcal{D}_{e}$ of $e$, and $v$ a boolean value. We say that $\rho$ validates $e[z]: v$, denoted by $\rho=e[z]: v$, if and only if

$$
\mathcal{E}(e)(\rho)[z]=v .
$$

By extension, we say that $\rho$ validates $e: v$ on subdomain $\mathcal{D}$ ( denoted by $\rho \models_{\mathcal{D}}$ $e: v$ ) if and only if

$$
\forall z \in \mathcal{D}, \rho \models e[z]: v .
$$

Definition 3.9 (Interpretation rules of $\models$ ). Boolean operators are interpreted as usual.

Definition 3.10 (Well-defined environment). We say that an environment $\rho$ is well-defined for a variable $X$ if for any index $z$ in its domain, $\rho(X)[z] \neq\{\perp, \top\}$. 


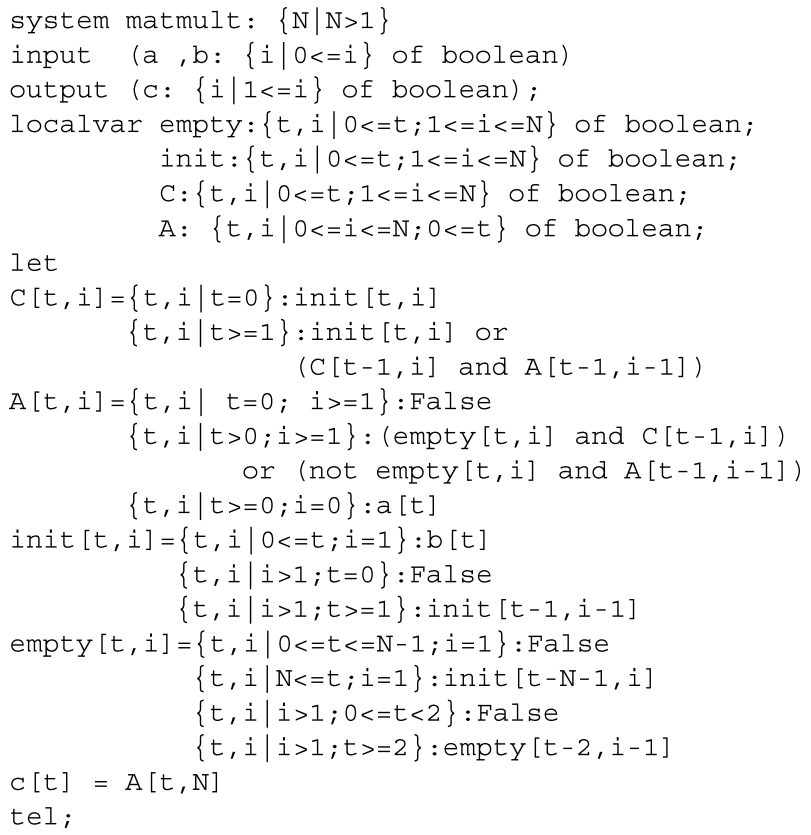

Fig. 3. Modeling matrix product system.

Definition 3.11 (Semantically correct environment). We say that an environment is semantically correct if it is well-defined and it is a least fixpoint for the system's semantics.

\subsection{The AlPha Language and the MMAlPha Environment}

3.5.1 Syntax of the Alpha Language. The AlphA language [Wilde 1994], originally developed for the design of regular (systolic) arrays, provides a syntax to define and manipulate SAREs. An ALPHA program is a mapping from input (polyhedral) variables to output variables defined by a SARE relating input, output and local variables. Each point in the domain of a local variable is uniquely defined by an affine recurrence equation. We present in Figure 3 an Alpha program corresponding to the matrix vector product.

In this example, expressions such as $\{t, i \mid 0<=i<=N ; 0<=t\}$ denote domains, $\mathrm{N}$ is a parameter, $\mathrm{a}, \mathrm{b}$ are the input variables, $\mathrm{c}$ is the output variable and empty, init, C, A are the local variables. Note that a program may have several output variables. Equations are displayed in array syntax form: instead of writing an equation as (for instance)

$$
\text { init }=\{t, i \mid i>1 ; t>=1\}: \text { init. }(t, i->t-1, i-1)
$$

we write it as

$$
\operatorname{init}[t, i]=\{t, i \mid i>1 ; t>=1\}: \text { init }[t-1, i-1] \text {. }
$$

Operators. The expressions appearing on the right-hand side of equations are obtained by combining variables or constants by means of two kinds of operators: pointwise operators and spatial operators. 
Pointwise operators are the classical data-parallel component wise generalizations of scalar operators. The domain of an expression defined by means of a pointwise operator is defined to be the intersection of the domains of the subexpressions combined by that operator.

Spatial operators are used to manipulate domains. We have three spatial operators, namely the dependence, restriction, and case operators.

- The dependence operator. Expressions such as $[t-2, i-1]$ denote dependencies functions. This notation just represents the dependency that maps $(t, i)$ to $(t-2, i-1)$.

-The restriction operator. It restricts the domain of an expression by means of affine constraints. For instance, the expression $\{t, i \mid i>1 ; t>=2\}$ : empty[t-2,i-1] restricts empty[t-2,i-1] to the intersection of the domain of empty $[t-2, i-1]$ with $\{t, i \mid i>1 ; t>=2\}$

- The case operator. It pieces together a set of disjoint subexpressions.

3.5.2 The MMAlpha Environment. The MMAlpha environment [Wilde 1993], based on Mathematica, implements a number of manipulations on ALPHA programs. This environment is used for high level synthesis: starting from a high-level (algorithmic) specification, the user refines it to an implementation under the form of VHDL code (for a hardware implementation), or C code (under the form of nested loops, for simulation or parallel execution), or a combination of both. All intermediate refinement steps consist in an ALPHA program that has been obtained from the previous one by a transformation rule. The whole process is semi-automatic, in the sense that transformations are automatic, but their sequencing has to be guided by the user. The environment provides a set of predefined commands and functions to namely achieve the following purposes.

—Static analysis of programs, including analysis of polyhedral domains' shapes and sizes.

- Simulation, architectural description, sequential code, or VHDL generation.

- Transformations of ALPHA programs, based on polyhedra manipulations and including pipelining of variables, scheduling, change of basis, and so on.

Most of these automatic transformations preserve the semantics of the initial specification, but the user may still introduce hand-tuned optimizations. This is the case in particular for control signals, or in the definition of interfaces between hardware and software parts of an implementation. This motivates the definition of verification methods to ensure the correctness of the implemented system.

\section{GENERAL IDEA}

Let $X$ be a multidimensional variable and $v$ a boolean value. Our aim is to prove that, for any well-defined environment $\rho, X$ will be equal to $v$ on a given domain. In this section, we will explain on an example the successive steps to achieve such a proof. 


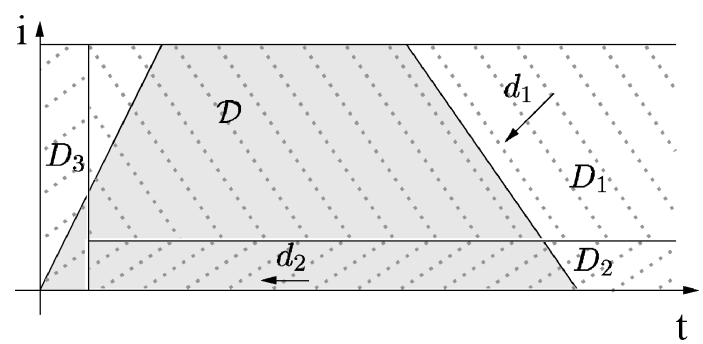

Fig. 4. Domains of variable $X$.

Let $X$ be a polyhedral variable defined on domain $\mathcal{D}_{X}=\mathcal{D}_{1} \cup \mathcal{D}_{2} \cup \mathcal{D}_{3}$ by the following equation:

$$
X=\left\{\begin{array}{l}
\mathcal{D}_{1}: X . d_{1} \wedge\left(Y . \delta_{1} \vee Y . \delta_{2}\right) \\
\mathcal{D}_{2}: X . d_{2} \\
\mathcal{D}_{3}: \text { True. }
\end{array}\right.
$$

We want to prove that $X$ is true on a given domain $\mathcal{D} \subset \mathcal{D}_{X}$. In Figure 4, we represent domain $\mathcal{D}_{X}$, and subdomains $\mathcal{D}_{1}, \mathcal{D}_{2}, \mathcal{D}_{3}$. Domain $\mathcal{D}$ is displayed in gray in this figure. Dependencies $d_{1}$ (for subdomain $\mathcal{D}_{1}$ ) and $d_{2}$ (for subdomain $\mathcal{D}_{2}$ ) are displayed as vectors.

The first step consists in using the value-substitution principle. This principle is based on a syntactic rewriting. We want to rewrite the expression defining $X$ into a semantically equivalent expression that does not any more depend on $X$. More formally, we want to find an expression $e$ such that

$$
\forall \rho, \quad \rho \models_{\mathcal{D}} X: v \Longleftrightarrow \rho \models_{\mathcal{D}} e: v .
$$

Let $\rho$ be an environment, and let us assume that $\rho \models_{\mathcal{D}} X: t t$. Thus, by definition of $\models$, all instances of $X$ on a point $z$ in $\mathcal{D}$ are equal to $t t$. Let us focus on expression $X . d_{1} \wedge\left(Y . \delta_{1} \vee Y . \delta_{2}\right)$ defined on domain $\mathcal{D}_{1}$. Let $z$ be a point of $\mathcal{D}_{1}$, two cases occur:

-if $d_{1}(z)$ is in $\mathcal{D}$, then instance $X\left[d_{1}(z)\right]$ is equal to $t$, and expression $X . d_{1} \wedge$ $\left(Y . \delta_{1} \vee Y . \delta_{2}\right)$ at point $z$ can be rewritten into $Y . \delta_{1} \vee Y . \delta_{2}$,

—otherwise, the expression is left unchanged.

For all points in $\left\{z \mid z \in d_{1}^{-1}(\mathcal{D}) \cap \mathcal{D}_{1}\right\}$, we thus can rewrite expression $X . d_{1} \wedge$ $\left(Y . \delta_{1} \vee Y . \delta_{2}\right)$ into $Y . \delta_{1} \vee Y . \delta_{2}$. We have the following equation:

$$
\mathcal{D}_{1}: X . d_{1} \wedge Y . \delta=\left\{\begin{array}{l}
d_{1}^{-1}(\mathcal{D}) \cap \mathcal{D}_{1}:\left(Y . \delta_{1} \vee Y . \delta_{2}\right) \\
\mathcal{D}_{1} \backslash d_{1}^{-1}(\mathcal{D}): X . d_{1} \wedge\left(Y . \delta_{1} \vee Y . \delta_{2}\right)
\end{array}\right.
$$

With a similar reasoning, we can rewrite expression $\mathcal{D}_{2}: X . d_{2}$ into the following equation.

$$
\mathcal{D}_{2}: X . d_{2}=\left\{\begin{array}{l}
d_{2}^{-1}(\mathcal{D}) \cap \mathcal{D}_{2}: \text { True } \\
\mathcal{D}_{2} \backslash d_{2}^{-1}(\mathcal{D}): X . d_{2} .
\end{array}\right.
$$




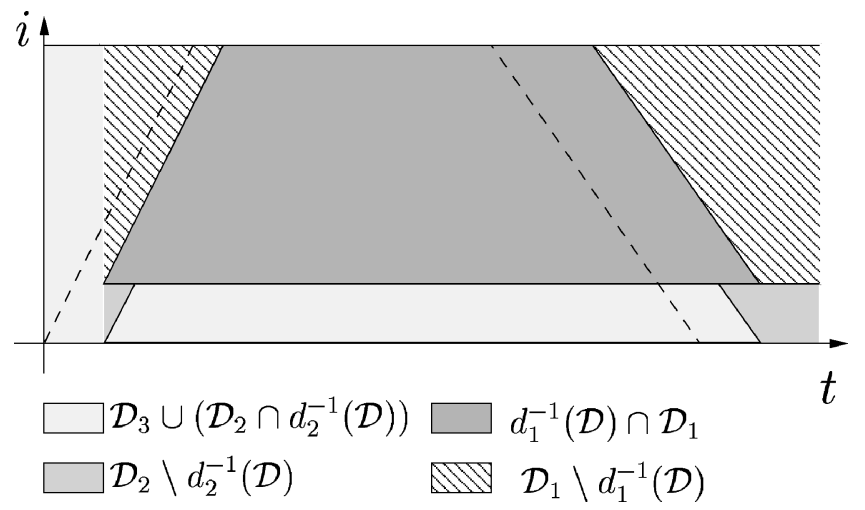

Fig. 5. Domains for variable $X$ after substitution.

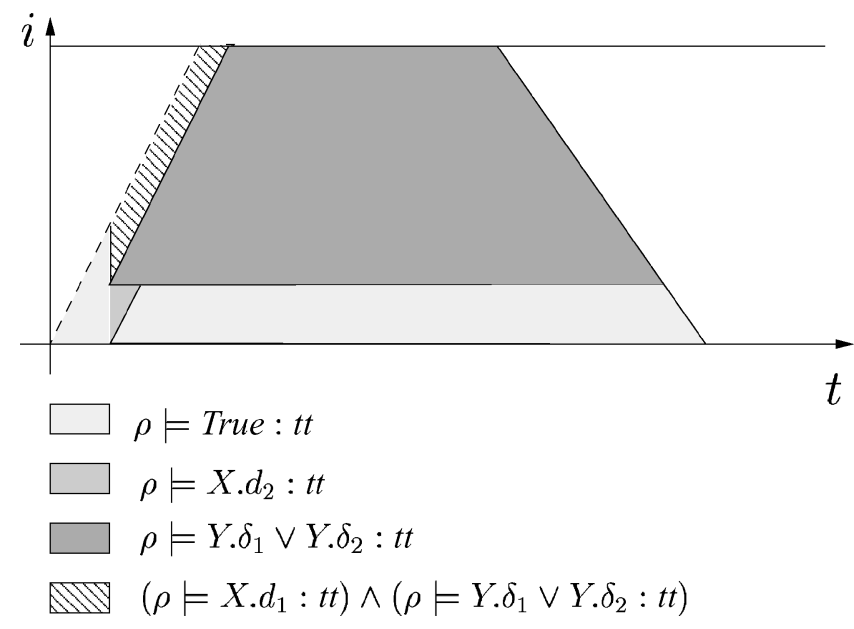

Fig. 6. Subgoals for variable $X$.

Thus, variable $X$ can be rewritten into

$$
X=\left\{\begin{array}{l}
\mathcal{D}_{1} \backslash d_{1}^{-1}(\mathcal{D}): X . d_{1} \wedge\left(Y . \delta_{1} \vee Y . \delta_{2}\right) \\
d_{1}^{-1}(\mathcal{D}) \cap \mathcal{D}_{1}: Y . \delta_{1} \vee Y . \delta_{2} \\
\mathcal{D}_{2} \backslash d_{2}^{-1}(\mathcal{D}): X . d_{2} \\
\mathcal{D}_{3} \cup\left(\mathcal{D}_{2} \backslash d_{2}^{-1}(\mathcal{D})\right): \text { True. }
\end{array}\right.
$$

All the domains involved in this equation are displayed in Figure 5. We have an equivalence between the initial equation of $X$ and the rewritten equation. The proof of this equivalence will be the purpose of the next section.

The proof is now split into four subgoals, corresponding to the newly generated subdomains. These subgoals are displayed in Figure 6.

-On domain $\left(\mathcal{D}_{3} \cup\left(\mathcal{D}_{2} \backslash d_{2}^{-1}(\mathcal{D})\right)\right) \cap \mathcal{D}$, we have the following subproof obligation:

$$
\rho=_{\left(\mathcal{D}_{3} \cup\left(\mathcal{D}_{2} \backslash d_{2}^{-1}(\mathcal{D})\right)\right) \cap \mathcal{D}} \text { True }: t t
$$


Thanks to the definition of $\models$, this subproof is immediately successful. -On domain $\left(\mathcal{D}_{2} \backslash d_{2}^{-1}(\mathcal{D})\right) \cap \mathcal{D}$, we have the following subproof obligation:

$$
\rho=_{\left(\mathcal{D}_{2} \backslash d_{2}^{-1}(\mathcal{D})\right) \cap \mathcal{D}} X . d_{2}: t t
$$

We consider two cases:

- either the domain $\left(\mathcal{D}_{2} \backslash d_{2}^{-1}(\mathcal{D})\right) \cap \mathcal{D}$ is empty, and thus there is no subproof obligation,

- or, as it is the case in our example, this domain is not empty. We are not able to directly prove that $X . d_{2}$ is true on domain $\mathcal{D}^{\prime}=\left(\mathcal{D}_{2} \backslash d_{2}^{-1}(\mathcal{D})\right) \cap \mathcal{D}$. Instead, we will prove a more general result, by widening domain $\mathcal{D}^{\prime}$. We use a heuristic approach to choose in which direction the domain has to be widened. In this particular example, $\mathcal{D}^{\prime}$ is widened along the direction defined by $d_{2}$. The widening domain $\mathcal{D}_{\text {wid }}$ is built from domain $\mathcal{D}^{\prime}$ as follows: let $z$ be in $\mathcal{D}_{\text {wid }}$, then two cases may occur:

- either $d_{2}(z) \in \mathcal{D}_{2}$, and $d_{2}(z) \in \mathcal{D}_{\text {wid }}$, - or $d_{2}(z) \notin \mathcal{D}_{2}$, and $d_{2}(z) \notin \mathcal{D}_{\text {wid }}$.

In our example, we then are able to prove that

$$
\rho \models_{\mathcal{D}_{\text {wid }}} X: t t
$$

-On domain $\left(d_{1}^{-1}(\mathcal{D}) \cap \mathcal{D}_{1}\right) \cap \mathcal{D}$, we have the following subproof obligation.

$$
\rho=_{\left(d_{1}^{-1}(\mathcal{D}) \cap \mathcal{D}_{1}\right) \cap \mathcal{D}} Y . \delta_{1} \vee Y . \delta_{2}: t t
$$

In this case, we add to the system a new variable $W$ defined on the domain of expression $Y . \delta_{1} \vee Y . \delta_{2}$, and the problem is turned into proving

$$
\rho \models_{\left(d_{1}^{-1}(\mathcal{D}) \cap \mathcal{D}_{1}\right) \cap \mathcal{D}} W: t t
$$

This will be done by recursively rewriting $W$. This is the purpose of the automatic invariant detection principle described in Section 6.

-On domain $\left(\mathcal{D}_{1} \backslash d_{1}^{-1}(\mathcal{D})\right) \cap \mathcal{D}$, we have the following subproof obligation:

$$
\rho \models_{\left(\mathcal{D}_{1} \backslash d_{1}^{-1}(\mathcal{D})\right) \cap \mathcal{D}} X . d_{1} \wedge\left(Y . \delta_{1} \vee Y . \delta_{2}\right): t t .
$$

Due to the interpretation of $\models$, this obligation is turned into

$$
\left(\rho \models_{\left(\mathcal{D}_{1} \backslash d_{1}^{-1}(\mathcal{D})\right) \cap \mathcal{D}} X . d_{1}: t t\right) \wedge\left(\rho \models_{\left(\mathcal{D}_{1} \backslash d_{1}^{-1}(\mathcal{D})\right) \cap \mathcal{D}} Y . \delta_{1} \vee Y . \delta_{2}: t t\right) .
$$

We thus generate two new subproof obligations:

—one concerning $\rho \models_{\left(\mathcal{D}_{1} \backslash d_{1}^{-1}(\mathcal{D})\right) \cap \mathcal{D}} X . d_{1}: t t$,

一the other one concerning $\rho=_{\left(\mathcal{D}_{1} \backslash d_{1}^{-1}(\mathcal{D})\right) \cap \mathcal{D}} Y . \delta_{1} \vee Y . \delta_{2}: t t$.

These two subproof obligations are of the same nature as for (4) and (5).

We have decomposed our initial goal into several proof obligations. The next sections explain how these proof obligations will be decomposed further, until we find constants or input variables in every branch. The whole process is finite, and we will sketch the proof of this fact in Section 8.

\section{PROVING WITH SUBSTITUTIONS}

In this section, we generalize and formalize the substitution mechanism seen in Section 4, prove its validity, and show how it is applied on a concrete example. Let $X$ be a polyhedral variable defined on a domain $\mathcal{D}_{X}$. Let $v$ be a boolean 
value, and $\mathcal{D}$ a sub-domain of $\mathcal{D}_{X}$. Let us assume that we want to prove that for any well-defined environment $\rho$,

$$
\rho \models_{\mathcal{D}} X: v .
$$

We want to find an expression $e$ such that for any well-defined environment $\rho$

$$
\rho \models_{\mathcal{D}} X \Longleftrightarrow \rho \models_{\mathcal{D}} e .
$$

In the previous section we have seen on an example how to compute $e$. The equivalence is given to us by the following informal argument. As we only consider systems which have been scheduled, we can proceed by induction on time. For any two instances $X[z]$ and $X\left[z^{\prime}\right]$ such that $X[z] \rightarrow X\left[z^{\prime}\right]$, we know that $X\left[z^{\prime}\right]$ will be computed before $X[z]$ in the given schedule. We then might assume that $X\left[z^{\prime}\right]$ is equal to $v$ in order to prove that $X[z]$ is equal to $v$. Intuitively, this allows us to substitute $X\left[z^{\prime}\right]$ by constant $v$ in the expression defining $X$. After this substitution step, the resulting expression is simplified according to a set of boolean simplification rules that preserve the semantics of expressions. In the following, we formalize this substitution principle and prove its validity.

\subsection{Value-Substitutions}

We first have to identify those index points which do not induce a recursive dependency for $X$ on domain $\mathcal{D}$.

Definition 5.1 (External Points). Let $X$ be a variable, $\mathcal{D}$ a domain such that $\mathcal{D} \subset \mathcal{D}_{X}$, and $\mathcal{D}^{\prime}$ the domain of a branch in the equation defining $X$. We call external points of $X$ for domain $\mathcal{D} \subset \mathcal{D}_{X}$ with respect to domain $\mathcal{D}^{\prime} \subset \mathcal{D}_{X}$ and the dependency $d$, denoted by $\mathcal{E P}_{d, \mathcal{D}^{\prime}, \mathcal{D}}$, the set of all points $z$ in $\mathcal{D}^{\prime}$ such that all variable instances $X$ depends on are neither instances of $X$, nor an instance of $X$ at point $d(z)$ in $\mathcal{D}$.

$$
\begin{array}{ll}
\mathcal{E P}_{d, \mathcal{D}^{\prime}, \mathcal{D}}= & \\
\left\{z \in \mathcal{D}^{\prime} \cap \mathcal{D} \mid \forall Y, \forall z^{\prime}, X[z] \rightarrow Y\left[z^{\prime}\right] \Longrightarrow\right. & Y \neq X \\
& \vee\left(Y=X \wedge z^{\prime} \neq d(z)\right) \\
& \left.\vee\left(Y=X \wedge z^{\prime}=d(z) \wedge z^{\prime} \notin \mathcal{D}\right)\right\} .
\end{array}
$$

Let us denote by $\mathcal{E} \mathcal{P}_{X, d, \mathcal{D}}$ (or, for short, $\mathcal{E} \mathcal{P}_{X}$ when no ambiguity may occur), the set of all external points $\mathcal{E} \mathcal{P}_{d, \mathcal{D}^{\prime}, \mathcal{D}}$ for domain $\mathcal{D}_{X}$.

Example. For the previous example in Section $4, \mathcal{E} \mathcal{P}_{d_{1}, \mathcal{D}_{1}, \mathcal{D}}$ and $\mathcal{E P}_{d_{2}, \mathcal{D}_{2}, \mathcal{D}}$ are defined by the following equations.

$$
\begin{aligned}
& \mathcal{E} \mathcal{P}_{d_{1}, \mathcal{D}_{1}, \mathcal{D}}=\left(\mathcal{D}_{1} \backslash d_{1}^{-1}(\mathcal{D})\right) \cap \mathcal{D} \\
& \mathcal{E} \mathcal{P}_{d_{2}, \mathcal{D}_{2}, \mathcal{D}}=\left(\mathcal{D}_{2} \backslash d_{2}^{-1}(\mathcal{D})\right) \cap \mathcal{D} .
\end{aligned}
$$

Domain $\mathcal{E} \mathcal{P}_{d_{1}, \mathcal{D}_{1}, \mathcal{D}}$ is displayed in dark gray in Figure 7.

The following lemma states the validity of the substitution of a variable by its value.

Lemma 5.2 (Substitution). Let $\rho$ be a well-defined environment, $g$ an arbitrary boolean function, and $v$ a boolean value. Thus, if $\rho$ validates $X . d: v$ 


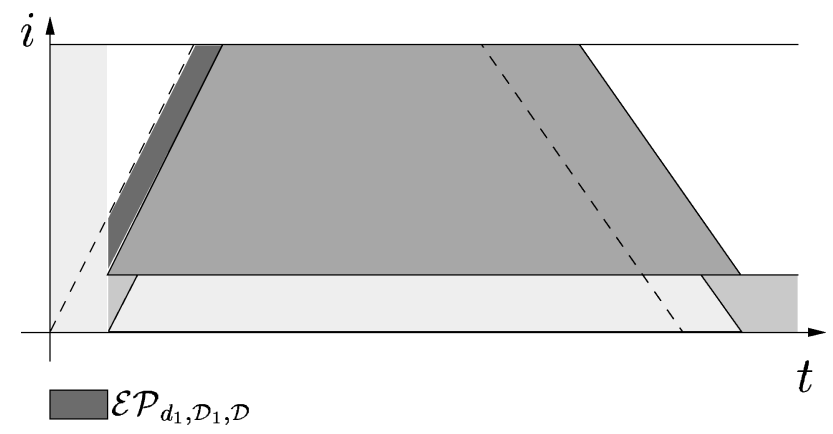

Fig. 7. Domain $\mathcal{E} \mathcal{P}_{d_{1}, \mathcal{D}_{1}, \mathcal{D}}$.

on a domain $\mathcal{D}$, expressions $g\left(\ldots, X . d_{i}, \ldots\right)$ and $g(\ldots, v, \ldots)$ are semantically equivalent for the environment $\rho$ on domain $\mathcal{D}$.

Proof. The proof is immediate, by definition of the semantics of expressions.

Let $d$ be a dependency of variable $X$, in the equation defining $X$. Let $e$ be the expression $g(\ldots, X . d, \ldots)$ in which every instance of the expression $X . d$ has been substituted by the constant $v$. We define a new variable $X^{\prime}$ by the following equation.

$$
X^{\prime}=\left\{\begin{array}{l}
\mathcal{E} \mathcal{P}_{X}: X \\
\mathcal{D}_{X} \backslash \mathcal{E} \mathcal{P}_{X}: e
\end{array}\right.
$$

Example. For the previous example, and for dependency $d_{1}$, we have

$$
X^{\prime}=\left\{\begin{array}{l}
\mathcal{D}_{1} \backslash d_{1}^{-1}(\mathcal{D}): X . d_{1} \wedge\left(Y . \delta_{1} \vee Y . \delta_{2}\right) \\
d_{1}^{-1}(\mathcal{D}) \cap \mathcal{D}_{1}:\left(Y . \delta_{1} \vee Y . \delta_{2}\right) \\
\mathcal{D}_{2}: X . d_{2} \\
\mathcal{D}_{3}: \text { True }
\end{array}\right.
$$

We can now prove equivalence (6).

PRoposition 5.2. Let $\rho$ be a semantically correct environment, $X^{\prime}$ be defined as above, and $v$ a value. Then,

$$
\rho \models \mathcal{D}_{X} X^{\prime}: v \Longleftrightarrow \rho \models_{\mathcal{D}_{X}} X: v .
$$

Proof. cf. Appendix A.

Until now, we have substituted all occurrences of $X . d$ for a single dependency $d$. We have to repeat the process for all dependencies $d^{\prime}$ appearing in the initial definition of $X$.

\subsection{Propositional Simplification}

After value-substitution, we get boolean expressions containing constants. In order to simplify them, we translate expressions into the Mathematica format and rely on the propositional simplification procedures of this tool. The resulting 
expressions are either single True or False constants, or boolean expressions involving other variables than $X$. These expressions are translated back into the ALPHA format.

Of course, this transformation is semantically correct: if $e$ is a boolean expression and $e^{\prime}$ is obtained from $e$ after propositional simplification, then for any environment $\rho$ and for any $z$ in $\mathcal{D}_{e}, \rho \models e[z]: v \Longleftrightarrow \rho \models e^{\prime}[z]: v$.

\subsection{Soundness}

From the results of previous sections, we directly can state the following theorem, that expresses the fact that $X$ is equal to a value $v$ on its entire domain as soon as it is equal to value $v$ on its external points, and its defining expression can be reduced to constants equal to $v$ in all branches.

Theorem 5.4 (Soundness of the VAlue-SUbSTITUTION PRINCIPLe). Let $X$ be a variable and $v$ a boolean value such that value-substitution and propositional simplification have been performed on each expression appearing on the rhs of the equation defining $X$. If on $\mathcal{D}_{X} \backslash \mathcal{E} \mathcal{P}_{X} X$ is now defined by constants equal to $v$ in all branches, then for any semantically correct environment $\rho$ such that $\rho \models_{\mathcal{E P}_{X}} X: v$, we have $\rho \models_{\mathcal{D}_{X}} X: v$.

\subsection{Application to the DLMS Filter}

Let us come back to the first example of Section 2. Variable $W \operatorname{ctl} 1 P$ is defined by the following equation on domain $\{t, p \mid p \leq t \leq p-N+M ; 0 \leq p \leq N-1\}$.

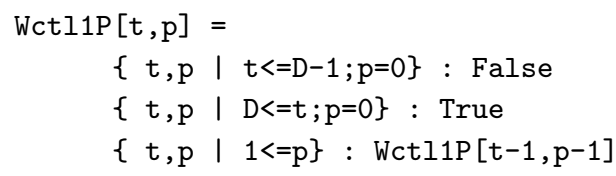

We have two properties to prove here. The first one is that on domain $\mathcal{D}_{1}=$ $\{t, p \mid p \leq t \leq p+D ; 0 \leq p \leq N-1\}, W c t l 1 P$ is false, the second one is that on domain $\mathcal{D}_{2}=\{t, p \mid p+D \leq t<p-N+M ; 0 \leq p \leq N-1\}$, Wctl1P is true. The value-substitution mechanism automatically yields constants since Wctl1P is substituted by ff on domain $\{t, p \mid p \leq t \leq p+D ; 0 \leq p \leq N-1\}$, and Wctl1P is substituted by $t t$ on domain $\{t, p \mid p+D \leq t<p-N+M ; 0 \leq p \leq N-1\}$. All this process is fully automatic.

\section{AUTOMATIC INVARIANT DETECTION}

The substitution mechanism succeeds when $X$ depends only on itself (or on initial values), or when the other variables $X$ depends on have been eliminated by propositional simplification rules. This is the case for many simple control signals, for instance when the signal is propagated without many modifications. In that case, $X$ can be seen as an invariant: if $X$ is equal to a value $v$ "at the beginning," then $X$ remains equal to $v$ "always and everywhere." For more complex control signals, we will have to look for more specific invariants. To restrict the set of formulae we investigate, we try to find an invariant by combining subexpressions appearing in the definitions of variables. 
Let us focus on a variable $P$ defined by the following equation, where $l_{P} \in$ $\mathbb{N} \backslash\{0\}$.

$$
P=\underset{i=1}{l_{P}} X_{i} \cdot d_{i}
$$

Let us assume that for any well-defined environment $\rho$, we have $\rho \models P: t t$. Let $W$ be a variable defined by the following equation, for $l_{W} \in \mathbb{N} \backslash\{0\}$, and for all $j$ in $\left\{1 \ldots l_{W}\right\}, l_{j} \in \mathbb{N} \backslash\{0\}$.

$$
W=\stackrel{l_{W}}{l_{j}} \underset{j=1}{\vee} X_{i=1}^{\prime} \cdot d_{i}^{\prime} .
$$

The idea is to use variable $P$ as a pattern to rewrite some subexpressions of the expression defining $W$. Since we know that $\rho \models P: t t$, we will thus be able to simplify $W$.

We will first show how to detect pattern $P$ in variable $W$, then we will see how we can apply this method for automatic invariant detection.

\subsection{Determining a Set of Candidates}

The idea is to substitute each subterm of $W$ that matches pattern $P$ by an occurrence of $P$ instantiated with the right dependencies.

Example. Let $P$ and $W$ be defined by the following equations.

$$
\begin{aligned}
P & =X . d_{1} \vee Y . d_{2} \\
W & =\left(X . \delta_{1} \vee X . \delta_{2} \vee Y . \delta_{3}\right) \wedge\left(X . \delta_{4} \vee W . \delta_{5}\right)
\end{aligned}
$$

We want to rewrite $W$ using pattern $P$. In other words, we want to find a dependency $d$ such that, for example

$$
W=P . d \wedge \ldots
$$

and such that, if we substitute $P$ by its definition in Eq. (10), we get Eq. (9).

We use the following notations.

$-T_{W}$ is the set of all disjunctions in $W$,

$-\tau$ denotes an element of $T_{W}$,

$-\operatorname{Var}(\tau)$ is the set of all pairs $(V, d)$ where $V$ is a variable and $d$ a dependency, such that $V . d$ appears in the definition of $\tau$,

- Used $(P)$ is the set of all pairs $(V, d)$ where $V$ is a variable and $d$ a dependency, such that $V . d$ appears in the definition of $P$.

Example. For the example above, we have the following definitions.

$$
\begin{aligned}
\operatorname{Used}(P) & =\left\{\left(X, d_{1}\right),\left(Y, d_{2}\right)\right\} \\
T_{W} & =\left\{\left(X . \delta_{1} \vee X . \delta_{2} \vee Y . \delta_{3}\right),\left(X . \delta_{4} \vee W . \delta_{5}\right)\right\} .
\end{aligned}
$$

Let us take $\tau$ equal to $X . \delta_{1} \wedge X . \delta_{2} \wedge Y . \delta_{3}$, then,

$$
\operatorname{Var}(\tau)=\left\{\left(X, \delta_{1}\right),\left(X, \delta_{2}\right),\left(Y, \delta_{3}\right)\right\} .
$$


We must find a dependency $d$ such that $P . d$ matches a subexpression of $\tau$. If such a dependency exists, then every variable appearing in $P$ appears in $\tau$. Thus, there must exist an injective application $\varphi$ from $U \operatorname{sed}(P)$ to $\operatorname{Var}(\tau)$, mapping any pair of $U \operatorname{sed}(P)$ to another pair in $\operatorname{Var}(\tau)$, such that the first component in both pairs is the same.

$$
\begin{aligned}
\varphi: \operatorname{Used}(P) & \rightarrow \operatorname{Var}(\tau) \\
(V, d) & \rightarrow\left(V, d^{\prime}\right) .
\end{aligned}
$$

Example. With the previously defined elements, we have two injective applications.

$$
\begin{aligned}
\varphi_{1}: & \left(X, d_{1}\right) \rightarrow\left(X, \delta_{1}\right) \varphi_{2}:\left(X, d_{1}\right) \rightarrow\left(X, \delta_{2}\right) \\
& \left(Y, d_{2}\right) \rightarrow\left(Y, \delta_{3}\right) \\
& \left(Y, d_{2}\right) \rightarrow\left(Y, \delta_{3}\right) .
\end{aligned}
$$

Let us assume that such an injective application $\varphi$ exists. By extension, we denote by $\varphi$, the application mapping $d$ to $d^{\prime}$ when $\varphi(V, d)=\left(V, d^{\prime}\right)$. We now have to compute a dependency $d$ such that $P . d$ is a pattern for a subexpression of $\tau$. That is, we have to find a dependency $d^{\prime \prime}$ such that for each $(V, d)$ in $\operatorname{Used}(P), d \circ d^{\prime \prime}=\varphi(d) \circ d$. We will have to solve the following system.

$$
\mathcal{S}(\varphi)=\cap_{(V, d) \in \operatorname{Used}(P)}\left\{d^{\prime \prime} \mid d \circ d^{\prime \prime}=\varphi(d) \circ d\right\} .
$$

In other words, $\mathcal{S}(\varphi)$ is the set of dependencies such that $P$ matches $\tau$.

\section{Example.}

$$
\begin{aligned}
& \mathcal{S}\left(\varphi_{1}\right)=\left\{d^{\prime \prime} \mid d_{1} \circ d^{\prime \prime}=\delta_{1}\right\} \cap\left\{d^{\prime \prime} \mid d_{2} \circ d^{\prime \prime}=\delta_{3}\right\} \\
& \mathcal{S}\left(\varphi_{2}\right)=\left\{d^{\prime \prime} \mid d_{1} \circ d^{\prime \prime}=\delta_{2}\right\} \cap\left\{d^{\prime \prime} \mid d_{2} \circ d^{\prime \prime}=\delta_{3}\right\} .
\end{aligned}
$$

Thus, if $d^{\prime \prime}$ is a dependency in $\mathcal{S}\left(\varphi_{1}\right)$, variable $W$ can be rewritten into the following equation.

$$
W=\left(X . \delta_{2} \vee P . d^{\prime \prime}\right) \wedge\left(X . \delta_{4} \vee W . \delta_{5}\right) .
$$

To find all dependencies that can match $\tau$, we do the union of $\mathcal{S}(\varphi)$ for all possible injections. Note that all the dependencies in $\mathcal{S}(\varphi)$ have the same dimensions. Since $(V, d)$ is in $U$ sed $(P)$, the expression $V . d$ has the same dimension as $P$. As a consequence, all the matrices representing the second component of a pair in $U \operatorname{sed}(P)$ have the same number of columns. We can conclude that every element of (11) shares the same number of rows. Similarly, as all the matrices representing $\varphi(d)$ share the same number of rows, every element of (11) shares the same number of columns.

\subsection{Solving A Dependencies System}

Solving one equation $d \circ d^{\prime \prime}=\varphi(d) \circ d$ in (11) is equivalent to finding a matrix $X$ such that $A X=B$, where $A$ and $B$ are known matrices. As we deal with integer matrices, $X$ must be solution to a linear Diophantine equation [Schrijver 1986]. The Polyhedral Library [Wilde 1993] gives an implementation of an algorithm solving such a system, based on the use of Hermite Normal Forms. Intersecting 
the solutions of a set of such systems is then done by constructing a compound matrix from the matrices of each equation (see Quinton et al. [1996] for more details).

\subsection{Application to Automatic Invariant Detection}

Let us remember that the substitution principle can be applied on a variable $W$ only when $W$ depends from itself. The idea is to use the pattern detection principle to rewrite $W$ such that it will depend from itself. Let us focus on a variable $W$ defined by the following equation. ${ }^{3}$

$$
W=\underset{i}{\vee} X_{i} \cdot d_{i} .
$$

This equation will be the pattern. We replace each variable on the rhs of this equation by its defining expression, and put the resulting expression into conjunctive normal form. We call $W_{\text {new }}$ the variable defined by this new equation. Then, we just have to detect if the pattern matches a subexpression of $W_{\text {new }}$. In this case, $W$ is now recursively defined.

Example. Let us come back to the example of Section 4. Let us recall that we had to prove that

$$
\rho \models_{\left(d_{1}^{-1}(\mathcal{D}) \cap \mathcal{D}_{1}\right) \cap \mathcal{D}} Y . \delta_{1} \vee Y . \delta_{2}: t t .
$$

We had introduced a new variable $W$ defined by $Y . \delta_{1} \vee Y . \delta_{2}$. Let us now assume that $Y$ is defined by the following equation.

$$
\begin{aligned}
& W=Y . \delta_{1} \vee Y . \delta_{2} \\
& Y=Y . \delta_{3} \vee Y . \delta_{4} .
\end{aligned}
$$

We substitute $Y$ by its definition in $W$, and we get the following equation.

$$
W_{\text {new }}=\mathcal{D}_{W}: Y . \delta_{3} \circ \delta_{1} \vee Y . \delta_{4} \circ \delta_{1} \vee Y . \delta_{3} \circ \delta_{2} \vee Y . \delta_{4} \circ \delta_{2} .
$$

We now apply the method of pattern detection with pattern $W$. We find two ways for $W$ to match $W_{\text {new }}$. Thus, $W$ (or $W_{\text {new }}$ ) is now defined by the following equation.

$$
W=W . \delta_{1} \vee W . \delta_{2} .
$$

The problem is now turned into proving that

$$
\rho \models_{\left(d_{1}^{-1}(\mathcal{D}) \cap \mathcal{D}_{1}\right) \cap \mathcal{D}} W: t t .
$$

On this problem, we can now apply the value-substitution principle. On a given subdomain of $\left.d_{1}^{-1}(\mathcal{D}) \cap \mathcal{D}_{1}\right) \cap \mathcal{D}$, we will obtain that $W$ is defined by True, other subdomains will be corresponding to input variables.

${ }^{3}$ If $W$ is defined by a conjunction, we just have to split it into separate subexpressions and treat them independently. 


\section{ITERATING THE PROCESS}

In the two previous sections, we saw two basic operations: value-substitution and invariant detection. We will iteratively combine these two operations. Two cases occur for the considered variable: either it is recursively defined, or it only depends on other variables.

-In the first case, we apply value-substitution. We thus get a variable whose defining expression involves constants (true or false), or boolean expressions depending on other variables. For each branch not defined by a constant, we add a new variable defined on the subdomain of the considered branch by the corresponding expression. This leads us to the second case.

- In the second case, we apply invariant computation (see Section 6). If we find an invariant, the variable is recursively defined and we come back to the first case. Otherwise, we will have to repeat the process.

We alternatively repeat both operations, and we stop when, after a valuesubstitution, either we find $a \neg v$ in one branch, or, in every branch, we have an input variable or the constant $v$.

We have to determine in which cases this iteration that alternates valuesubstitution and invariant computation will stop. The polyhedral model in its full generality handles recurrence equations with affine dependency functions. In the following, we restrict ourselves to uniform dependency functions, that is translations on indices. Note however that this restriction is not a too strong one since any affine recurrence equation can be rewritten into a uniform one by introducing new variables [Manjunathaiah et al. 2001]. With this restriction we will be able to characterize those cases when the iteration will be finite, and those when the iteration will be infinite.

Let us consider that we want to detect an invariant in the equation defining a variable $W$. Let $v$ be a boolean value. We want to prove, for any environment $\rho$

$$
\rho \models W: v .
$$

Variable $W$ is defined by means of only one boolean operator (otherwise we can split the proof into several subproof obligations). In a first time, we also assume that $W$ is defined by occurrences of only one variable. Intuitively, the problem of detecting an invariant if $W$ is defined by occurrences of several variables is not decidable in the general case, since there is no reason distinct variables would be defined by expressions sharing the same structure or the same dependencies. Thus, let us assume that $W$ is defined by the following equation,

$$
W=\underset{i}{\vee} X \cdot d_{i}
$$

If $v$ is the $f f$ value, we just have to prove that for all $i, \rho \models X . d_{i}:$ ff. If $v$ is the $t t$ value, we need to apply the invariant computation. In the rest of this paper, we will assume that $W$ is defined by a disjunction and that $V$ is the $t t$ value. We can extend our results to their dual counterparts by simply replacing each true value by a false value, each conjunction by a disjunction, and conversely. In this paper, we will first study termination on a base case consisting of variables 
defined by expressions involving selfdependencies, and composed of only one branch. Then, we will study termination in the case of variables defined by several equations. In those cases we study here, we show below that we are able to predict if our iterating process will stop, and if we will find an invariant.

\subsection{Case of a Single Branch}

We first restrict ourselves to the simplest case: the variable $X$ is defined by an expression involving only occurrences of $X$ (selfdependencies) and composed of only one branch and one boolean operator. We address the following problem: can we alternatively repeat automatic invariant generation and valuesubstitution until we discharge all proof obligations?

At this point, we assume that $d_{i}$ are all distinct, as the case of equality has been eliminated by propositional simplification.

Theorem 7.1. Let $W$ and $X$ be two polyhedral variables. Let us assume that we want to prove that for all environment $\rho, \rho \models W: t t$. Then, if $W$ is defined by the following equations,

$$
W=\underset{i}{\vee} X \cdot d_{i}
$$

we have,

-if $X$ is defined by $X=\vee_{j} X . d_{j}^{\prime}$, iterating alternatively automatic invariant generation and value-substitution is finite,

- if $X$ is defined by $=\wedge_{j} X . d_{j}^{\prime}$, iterating alternatively automatic invariant generation and value-substitution is infinite.

We will sketch the proof of Theorem 7.1 on examples.

Case $X=\vee_{j} X . d_{j}^{\prime}$. Let us assume that $X$ is defined by a disjunction of two terms.

$$
X=X . \delta_{1} \vee X . \delta_{2}
$$

We substitute $X$ in the definition of $W$ and we get after distribution of $\vee$ operators

$$
W=X . \delta_{1} \circ d_{1} \vee X . \delta_{2} \circ d_{1} \vee X . \delta_{1} \circ d_{2} \vee X . \delta_{2} \circ d_{2}
$$

Looking for an invariant resumes to finding a solution for one of six systems of equations on dependencies. If such a system has a solution $f$, we will be able to write $W=W . f \vee X . \delta_{i} \circ d_{j} \vee X . \delta_{i^{\prime}} \circ d_{j^{\prime}}$ for some $i, i^{\prime}, j, j^{\prime}$. After valuesubstitution, we will get $W=v$. Thus, we just need one solution to stop the iteration. Let us now show that, since we have uniform dependencies, there will always be a solution for at least one of the six systems. The following lemma considers one of the six systems.

Lemma 7.2. Let $d_{1}, d_{2}, \delta, \delta^{\prime}$ be uniform dependencies. The system

$$
S=\left\{\begin{array}{l}
\delta \circ d_{1}=d_{1} \circ f \\
\delta^{\prime} \circ d_{2}=d_{2} \circ f
\end{array}\right.
$$

ACM Transactions on Embedded Computing Systems, Vol. 4, No. 2, May 2005. 


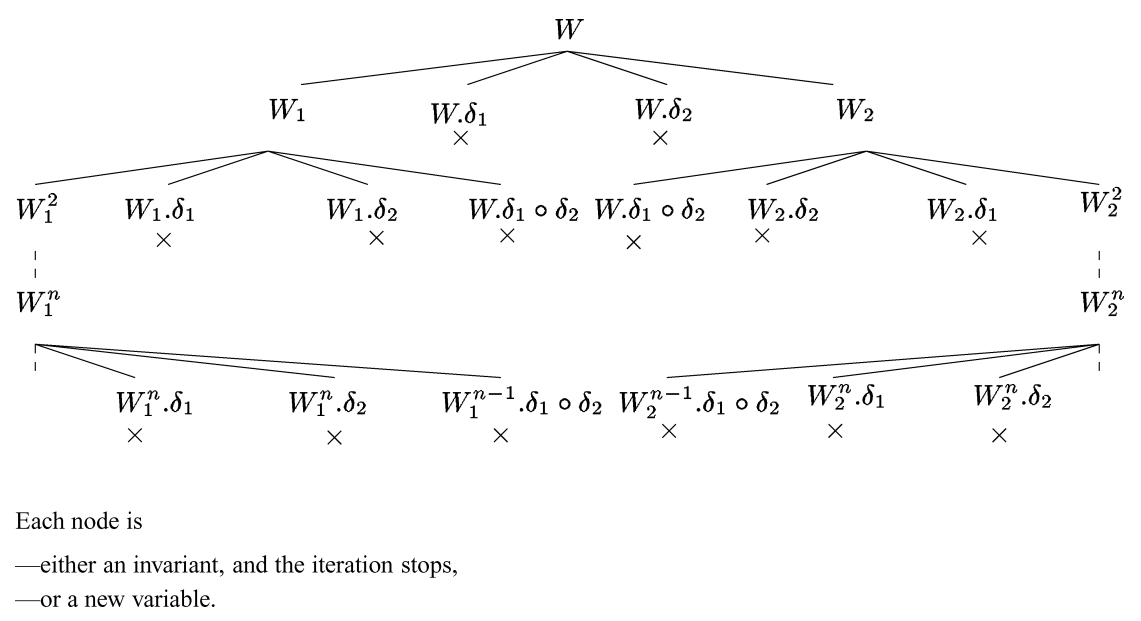

Fig. 8. Tree of invariants generation.

has a solution if and only if $\delta=\delta^{\prime}$. In this case, it has exactly one solution, namely $f=\delta$.

Proof. Since dependencies are uniform, the product is commutative.

We can apply this lemma to two out of the six systems (e.g. system $S$ ) and we conclude that we are sure to find two invariants. Thus, if $X$ is a disjunction, we are sure to stop the iteration.

The generalization to $n$ terms is immediate.

Case $X=\wedge_{j} X . d_{j}^{\prime}$. Let us now assume that $X$ is defined by a conjunction of two terms (the generalization to $n$ terms is immediate): $X=X . \delta_{1} \wedge X . \delta_{2}$. We substitute $X$ by its definition in $W$ and we get

$$
\begin{aligned}
W= & \left(X . \delta_{1} \circ d_{1} \vee X . \delta_{1} \circ d_{2}\right) \\
& \wedge\left(X . \delta_{2} \circ d_{1} \vee X . \delta_{2} \circ d_{2}\right) \\
& \wedge\left(X . \delta_{1} \circ d_{1} \vee X . \delta_{2} \circ d_{2}\right) \\
& \wedge\left(X \delta_{2} \circ d_{1} \vee X . \delta_{1} \circ d_{2}\right)
\end{aligned}
$$

Since $W$ is a conjunction, we now have to find an invariant in each subterm if we want to stop the iteration. Thanks to Lemma 7.2, we find an invariant in the first two subterms $\left(W . \delta_{1}\right.$ and $W . \delta_{2}$ (cf. Figure 8$)$ ). For the last two subterms, we now show that we cannot find any invariant using this method. Let $W_{1}$ and $W_{2}$ be defined respectively by $W_{1}=X . \delta_{1} \circ d_{1} \vee X . \delta_{2} \circ d_{2}$ and $W_{2}=X . \delta_{2} \circ d_{1} \vee X . \delta_{1} \circ$ $d_{2}$. For each variable $W_{1}$ and $W_{2}$, we have two systems to solve. If we have no solution, we substitute $X$ by its definition and we get

$$
\begin{array}{rlrl}
W_{1}= & \left(X . \delta_{1} \circ \delta_{1} \circ d_{1} \vee X . \delta_{1} \circ \delta_{2} \circ d_{2}\right) & W_{2}= & \left(X . \delta_{1} \circ \delta_{1} \circ d_{2} \vee X . \delta_{1} \circ \delta_{2} \circ d_{1}\right) \\
& \wedge\left(X . \delta_{2} \circ \delta_{1} \circ d_{1} \vee X . \delta_{2} \circ \delta_{2} \circ d_{2}\right) & \wedge\left(X . \delta_{2} \circ \delta_{1} \circ d_{2} \vee X . \delta_{2} \circ \delta_{2} \circ d_{1}\right) \\
& \wedge\left(X . \delta_{1} \circ \delta_{1} \circ d_{1} \vee X . \delta_{2} \circ \delta_{2} \circ d_{2}\right) & \wedge\left(X . \delta_{1} \circ \delta_{1} \circ d_{2} \vee X . \delta_{2} \circ \delta_{2} \circ d_{1}\right) \\
& \wedge\left(X . \delta_{2} \circ \delta_{1} \circ d_{1} \vee X . \delta_{1} \circ \delta_{2} \circ d_{2}\right) & & \wedge\left(X . \delta_{2} \circ \delta_{1} \circ d_{2} \vee X . \delta_{1} \circ \delta_{2} \circ d_{1}\right)
\end{array}
$$

Again, for the first two subterms and the last one of $W_{1}$ (respectively $W_{2}$ ), we find an invariant thanks to Lemma 7.2, but the problem again arises for the 
third subterm of $W_{1}$ (respectively $W_{2}$ ). We add variables for both third subterms. By iterating this process (cf. Figure 8), we successively add variables $W_{1}^{n}$ and $W_{2}^{n}$ defined by

$$
\begin{aligned}
& W_{1}^{n}=X . \delta_{1}^{n} \circ d_{1} \vee X . \delta_{2}^{n} \circ d_{2} \\
& W_{2}^{n}=X . \delta_{1}^{n} \circ d_{2} \vee X . \delta_{2}^{n} \circ d_{1} .
\end{aligned}
$$

For both variables, we look for pattern $W$. We get the four systems ${ }^{4}$ described in Lemma (B.1) in Appendix. With this Lemma, we show that in the best case, we will find an invariant in one branch, but for the other one the iteration will never stop.

Case of Negations. When negation operators appear in the definition of $W$, we apply the same techniques and get the following result.

Theorem 7.3. Let $W$ and $X$ be two polyhedral variables. Let us assume that we want to prove that for all environment $\rho, \rho \models W: t$. Then, if $W$ and $X$ are defined by the following equations, where $l_{1}, l_{2}, l \in \mathbb{N} \backslash 0$ and $\forall i \in\{1 . . l\}, m_{i} \in \mathbb{N}$,

$$
\begin{aligned}
W & =\left(\underset{j=1}{\vee} X . d_{j}\right) \vee\left(\underset{j=1}{l_{1}} \neg X \cdot d_{j}^{\prime}\right) \\
X & =\underset{i=1}{l_{2} m_{i}} \underset{j=1}{\vee} X \cdot d_{j}^{i}
\end{aligned}
$$

we have,

-iterating alternatively automatic invariant generation and value-substitution is finite, when

- either $l_{1}=l_{2}=1$,

$$
\begin{aligned}
& W=X \cdot d_{1} \vee \neg X \cdot d_{1}^{\prime} \\
& X=\stackrel{l}{\wedge}{\underset{m}{i}}_{j=1}^{\vee} X \cdot d_{j}^{i}
\end{aligned}
$$

-or $l_{1}=1 \wedge l_{2}>1 \wedge \forall i, m_{i}=1$,

$$
\begin{aligned}
& W=X . d_{1} \vee\left(\underset{j=1}{l_{2}} \neg X . d_{j}^{\prime}\right) \\
& X=\stackrel{{ }_{i=1}}{\wedge} X . d_{i}
\end{aligned}
$$

$\operatorname{or}_{1}>1 \wedge l_{2}=1 \wedge l=1$

$$
\begin{aligned}
W & \left.=\underset{j=1}{l_{1}} X . d_{j}\right) \vee \neg X . d_{1}^{\prime} \\
X & =\underset{j=1}{m_{i}} X . d_{j}
\end{aligned}
$$

\footnotetext{
${ }^{4}$ In fact, we must look for all patterns $W_{1}^{i}$ and $W_{2}^{j}$, but as for $W$ we will not find an invariant in every branch.

ACM Transactions on Embedded Computing Systems, Vol. 4, No. 2, May 2005.
} 
- for all the other cases, iterating alternatively automatic invariant generation and value-substitution is infinite.

\subsection{Case of Multiple Branches}

Let us now consider a variable defined by several branches. Without loss of generality, we restrict the problem to domains that are polyhedra and not unions of polyhedra. ${ }^{5}$ Let $\mathcal{D}_{1}$ and $\mathcal{D}_{2}$ be two domains whose intersection is empty. Let $W$ and $X$ be two variables defined by the following equations.

$$
\begin{aligned}
& W=\mathcal{D}_{W}: X . d_{1} \vee X . d_{2} \\
& X=\left\{\begin{array}{c}
\mathcal{D}_{1}: X . \delta_{1} \\
\mathcal{D}_{2}: X . \delta_{2} .
\end{array}\right.
\end{aligned}
$$

The first step consists in substituting $X$ by its definition in $W$. This yields the following recurrence equation.

$$
W=\left\{\begin{array}{l}
\mathcal{D}_{W} \cap d_{1}^{-1}\left(\mathcal{D}_{1}\right) \cap d_{2}^{-1}\left(\mathcal{D}_{1}\right): X . \delta_{1} \circ d_{1} \vee X . \delta_{1} \circ d_{2} \\
\mathcal{D}_{W} \cap d_{1}^{-1}\left(\mathcal{D}_{2}\right) \cap d_{2}^{-1}\left(\mathcal{D}_{2}\right): X . \delta_{2} \circ d_{1} \vee X . \delta_{2} \circ d_{2} \\
\mathcal{D}_{W} \cap d_{1}^{-1}\left(\mathcal{D}_{1}\right) \cap d_{2}^{-1}\left(\mathcal{D}_{2}\right): X . \delta_{1} \circ d_{1} \vee X . \delta_{2} \circ d_{2} \\
\mathcal{D}_{W} \cap d_{1}^{-1}\left(\mathcal{D}_{2}\right) \cap d_{2}^{-1}\left(\mathcal{D}_{1}\right): X . \delta_{2} \circ d_{1} \vee X . \delta_{1} \circ d_{2} .
\end{array}\right.
$$

Since dependencies are uniform, we know that there is an invariant in the first two branches, and we get

$$
W=\left\{\begin{array}{l}
\mathcal{D}_{W} \cap d_{1}^{-1}\left(\mathcal{D}_{1}\right) \cap d_{2}^{-1}\left(\mathcal{D}_{1}\right): W . \delta_{1} \\
\mathcal{D}_{W} \cap d_{1}^{-1}\left(\mathcal{D}_{2}\right) \cap d_{2}^{-1}\left(\mathcal{D}_{2}\right): W . \delta_{2} \\
\mathcal{D}_{W} \cap d_{1}^{-1}\left(\mathcal{D}_{1}\right) \cap d_{2}^{-1}\left(\mathcal{D}_{2}\right): X . \delta_{1} \circ d_{1} \vee X . \delta_{2} \circ d_{2} \\
\mathcal{D}_{W} \cap d_{1}^{-1}\left(\mathcal{D}_{2}\right) \cap d_{2}^{-1}\left(\mathcal{D}_{1}\right): X . \delta_{2} \circ d_{1} \vee X . \delta_{1} \circ d_{2} .
\end{array}\right.
$$

We have two branches where we find no invariant (Lemma 7.2). We may add variables: we will finally get a solution only if we manage to prove that, after a finite number of substitutions, we have an invariant in every branch.

As in the previous case when $X=\wedge_{i} X . d_{i}^{\prime}$, we will not find an invariant for every branch. The solution is to study the domain of added variables. We will prove that domains of these variables become empty after a finite number of iterations. Thus, it will not be necessary to find an invariant in every branch.

The idea is to project dependencies and domains on a vector $\vec{\omega}$ orthogonal to the hyperplane $P$ that separates domains $\mathcal{D}_{1}$ and $\mathcal{D}_{2}$ (cf. Figure 9 ). A domain $\mathcal{D}$ is not empty if and only if all the projections of $\mathcal{D}$ along all space dimensions are not empty. Let $\mathcal{D}^{\prime}$ be the intersection of $d_{1}^{-1}\left(\mathcal{D}_{1}\right)$ and $d_{2}^{-1}\left(\mathcal{D}_{2}\right)$. The preimage of $\mathcal{D}_{1}$ by one of the dependencies is a translation of $\mathcal{D}_{1}$ : the hyperplane $P$ delimiting $\mathcal{D}_{1}$ is translated, and its preimage $P_{1}$ delimits the preimage of $\mathcal{D}_{1}$. The same occurs for $\mathcal{D}_{2}$, preimage of which by $d_{2}$ is delimited by hyperplane $P_{2}$, preimage of $P$ by $d_{2}$. Thus, $\mathcal{D}^{\prime}$ is delimited by two hyperplanes $P_{1}$ and $P_{2}$ parallel to $P$ (cf. Figure 10), and the projection of $\mathcal{D}^{\prime}$ along any vector of $P$ is not empty. To

${ }^{5}$ If domains are unions of polyhedra, we split them into several branches in order to get single polyhedra. 


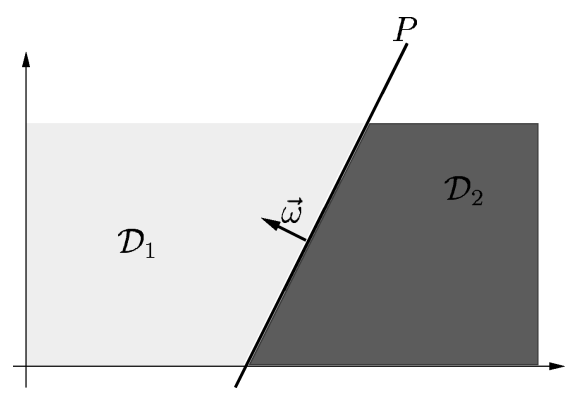

Fig. 9. Domains for a variable $X$ defined by two branches.

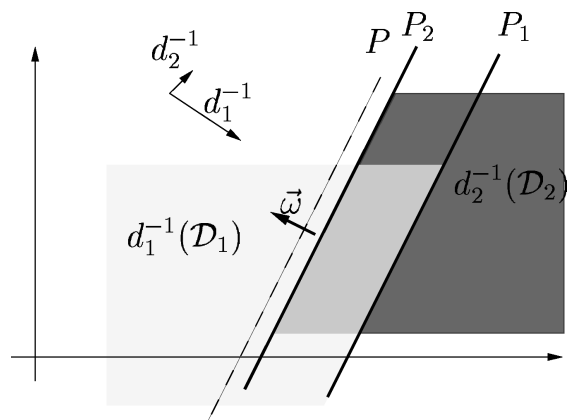

Fig. 10. Intersection of preimages $d_{1}^{-1}\left(\mathcal{D}_{1}\right)$ and $d_{2}^{-1}\left(\mathcal{D}_{2}\right)$.

prove the emptiness of $\mathcal{D}^{\prime}$, we thus need to prove that its projection along $\vec{\omega}$ (orthogonal to $P$ ) is empty.

Let us denote by $\alpha_{1}$ (respectively $\alpha_{2}$ ) the projection of $d_{1}$ (respectively $d_{2}$ ) along $\vec{\omega}$. As $d_{1} \neq d_{2}$, we know that $\alpha_{1} \neq \alpha_{2}$. We note that, depending on the respective values of $\alpha_{1}$ and $\alpha_{2}$, only one of the two intersections $d_{1}^{-1}\left(\mathcal{D}_{1}\right) \cap$ $d_{2}^{-1}\left(\mathcal{D}_{2}\right)$ and $d_{2}^{-1}\left(\mathcal{D}_{1}\right) \cap d_{1}^{-1}\left(\mathcal{D}_{2}\right)$ will not be empty. The case $\alpha_{2}<\alpha_{1}$ is illustrated in Figure 11, the case $\alpha_{1}<\alpha_{2}$ being symmetric.

Let us assume that we add a variable for the third branch of $W$, domain of which is not empty.

$$
Z_{1}=\mathcal{D}_{W} \cap d_{1}^{-1}\left(\mathcal{D}_{1}\right) \cap d_{2}^{-1}\left(\mathcal{D}_{2}\right): X . \delta_{1} \circ d_{1} \vee X . \delta_{2} \circ d_{2} .
$$

We substitute $X$ by its definition in Eq. (12). If we denote by $\mathcal{D}_{Z_{1}}$ the domain $\mathcal{D}_{W} \cap d_{1}^{-1}\left(\mathcal{D}_{1}\right) \cap d_{2}^{-1}\left(\mathcal{D}_{2}\right)$, we have

$$
Z_{1}=\left\{\begin{array}{l}
\mathcal{D}_{Z_{1}} \cap d_{1}^{-1} \circ \delta_{1}^{-1}\left(\mathcal{D}_{1}\right) \cap d_{2}^{-1} \circ \delta_{2}^{-1}\left(\mathcal{D}_{1}\right): Z_{1} . \delta_{1} \\
\mathcal{D}_{Z_{1}} \cap d_{1}^{-1} \circ \delta_{1}^{-1}\left(\mathcal{D}_{2}\right) \cap d_{2}^{-1} \circ \delta_{2}^{-1}\left(\mathcal{D}_{2}\right): Z_{1} . \delta_{2} \\
\mathcal{D}_{Z_{1}} \cap d_{1}^{-1} \circ \delta_{1}^{-1}\left(\mathcal{D}_{1}\right) \cap d_{2}^{-1} \circ \delta_{2}^{-1}\left(\mathcal{D}_{2}\right): X . \delta_{1} \circ \delta_{1} \circ d_{1} \vee X . \delta_{2} \circ \delta_{2} \circ d_{2} \\
\mathcal{D}_{Z_{1}} \cap d_{1}^{-1} \circ \delta_{1}^{-1}\left(\mathcal{D}_{2}\right) \cap d_{2}^{-1} \circ \delta_{2}^{-1}\left(\mathcal{D}_{1}\right): W . \delta_{2} \circ \delta_{1}
\end{array}\right.
$$

Let us note that we find no invariant in the third branch of the expression defining $Z_{1}$. Moreover, similarly as for $W$ before, one of the last two domains is empty. If it is domain $\mathcal{D}_{Z_{1}} \cap d_{1}^{-1} \circ \delta_{1}^{-1}\left(\mathcal{D}_{1}\right) \cap d_{2}^{-1} \circ \delta_{2}^{-1}\left(\mathcal{D}_{2}\right)$, the iteration ends up. In the other case, we still need to add a new variable and repeat the invariant detection. We need to prove that this process is finite. Let $q$ be an integer and 


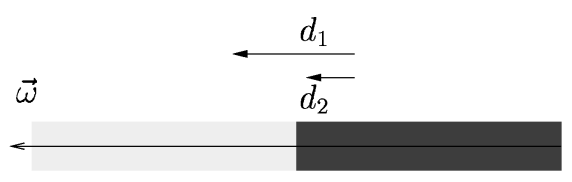

(a) Projection of domains $\mathcal{D}_{1}$ and $\mathcal{D}_{2}$ on $\vec{\omega}$.

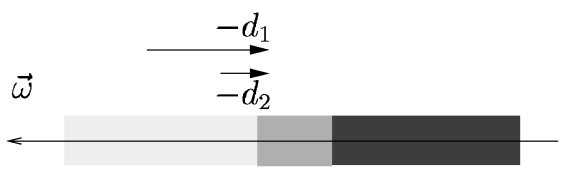

(b) $d_{1}^{-1}\left(\mathcal{D}_{1}\right) \cap d_{2}^{-1}\left(\mathcal{D}_{2}\right) \neq \emptyset$

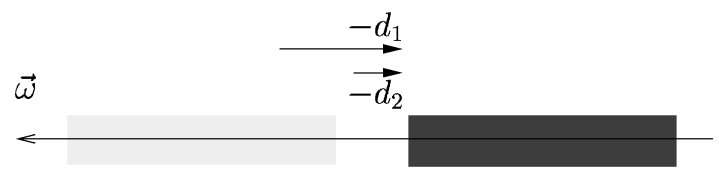

(c) $d_{2}^{-1}\left(\mathcal{D}_{1}\right) \cap d_{1}^{-1}\left(\mathcal{D}_{2}\right)=\emptyset$

Fig. 11. Projection of domains $\mathcal{D}_{1}$ and $\mathcal{D}_{2}$ on $\vec{\omega}$, and projection of their preimages in the case $\alpha_{2}<\alpha_{1}$.

$Z_{q}$ the variable we add at step $q$. This variable is defined on domain $\mathcal{D}_{Z_{q}}=$ $\bigcap_{i=0}^{q-1} d_{1}^{-1} \circ\left(\delta_{1}^{-1}\right)^{i}\left(\mathcal{D}_{1}\right) \cap d_{2}^{-1} \circ\left(\delta_{2}^{-1}\right)^{i}\left(\mathcal{D}_{2}\right)$ by

$$
Z_{q}=\mathcal{D}_{Z_{q}}: X . \delta_{1}{ }^{q} \circ d_{1} \vee X . \delta_{2}{ }^{q} \circ d_{2} .
$$

We substitute $X$ by its definition and we get

$Z_{q}=\left\{\begin{array}{l}\mathcal{D}_{Z_{q}} \cap d_{1}^{-1} \circ\left(\delta_{1}^{-1}\right)^{q}\left(\mathcal{D}_{1}\right) \cap d_{2}^{-1} \circ\left(\delta_{2}^{-1}\right)^{q}\left(\mathcal{D}_{1}\right): X . \delta_{1} \circ \delta_{1}{ }^{q} \circ d_{1} \vee X . \delta_{1} \circ \delta_{2}{ }^{q} \circ d_{2} \\ \mathcal{D}_{Z_{q}} \cap d_{1}^{-1} \circ\left(\delta_{1}^{-1}\right)^{q}\left(\mathcal{D}_{2}\right) \cap d_{2}^{-1} \circ\left(\delta_{2}^{-1}\right)^{q}\left(\mathcal{D}_{2}\right): X . \delta_{2} \circ \delta_{1}{ }^{q} \circ d_{1} \vee X . \delta_{2} \circ \delta_{2}{ }^{q} \circ d_{2} \\ \mathcal{D}_{Z_{q}} \cap d_{1}^{-1} \circ\left(\delta_{1}^{-1}\right)^{q}\left(\mathcal{D}_{1}\right) \cap d_{2}^{-1} \circ\left(\delta_{2}^{-1}\right)^{q}\left(\mathcal{D}_{2}\right): X . \delta_{1} \circ \delta_{1}{ }^{q} \circ d_{1} \vee X . \delta_{2} \circ \delta_{2}{ }^{q} \circ d_{2} \\ \mathcal{D}_{Z_{q}} \cap d_{1}^{-1} \circ\left(\delta_{1}^{-1}\right)^{q}\left(\mathcal{D}_{2}\right) \cap d_{2}^{-1} \circ\left(\delta_{2}^{-1}\right)^{q}\left(\mathcal{D}_{1}\right): X . \delta_{2} \circ \delta_{1}{ }^{q} \circ d_{1} \vee X . \delta_{1} \circ \delta_{2}{ }^{q} \circ d_{2} .\end{array}\right.$

We look for an invariant in every branch and we get

$$
Z_{q}=\left\{\begin{array}{l}
\mathcal{D}_{Z_{q}} \cap d_{1}^{-1} \circ\left(\delta_{1}^{-1}\right)^{q}\left(\mathcal{D}_{1}\right) \cap d_{2}^{-1} \circ\left(\delta_{2}^{-1}\right)^{q}\left(\mathcal{D}_{1}\right): Z_{q} . \delta_{1} \\
\mathcal{D}_{Z_{q}} \cap d_{1}^{-1} \circ\left(\delta_{1}^{-1}\right)^{q}\left(\mathcal{D}_{2}\right) \cap d_{2}^{-1} \circ\left(\delta_{2}^{-1}\right)^{q}\left(\mathcal{D}_{2}\right): Z_{q} . \delta_{2} \\
\mathcal{D}_{Z_{q}} \cap d_{1}^{-1} \circ\left(\delta_{1}^{-1}\right)^{q}\left(\mathcal{D}_{1}\right) \cap d_{2}^{-1} \circ\left(\delta_{2}^{-1}\right)^{q}\left(\mathcal{D}_{2}\right): X . \delta_{1}{ }^{q+1} \circ d_{1} \vee X . \delta_{2}{ }^{q+1} \circ d_{2} \\
\mathcal{D}_{Z_{q}} \cap d_{1}^{-1} \circ\left(\delta_{1}^{-1}\right)^{q}\left(\mathcal{D}_{2}\right) \cap d_{2}^{-1} \circ\left(\delta_{2}^{-1}\right)^{q}\left(\mathcal{D}_{1}\right): Z_{q-1} . \delta_{1} \circ \delta_{2} .
\end{array}\right.
$$

We represent in Figure 12, the set of all added variables. At each step, we find an invariant in three branches but not in the last one. To ensure the termination of this process, we need to show that there exists a value of $q$ such that $\mathcal{D}_{Z_{q}} \cap$ $d_{1}^{-1} \circ\left(\delta_{1}^{-1}\right)^{q}\left(\mathcal{D}_{1}\right) \cap d_{2}^{-1} \circ\left(\delta_{2}^{-1}\right)^{q}\left(\mathcal{D}_{2}\right)$ is empty.

At each step, we compute the preimages of $\mathcal{D}_{1}$ and $\mathcal{D}_{2}$ by, respectively, $d_{1}^{-1}$ 。 $\left(\delta_{1}^{-1}\right)^{q}$ and $d_{2}^{-1} \circ\left(\delta_{2}^{-1}\right)^{q}$, we project them along $\vec{\omega}$ and we compute the intersection 


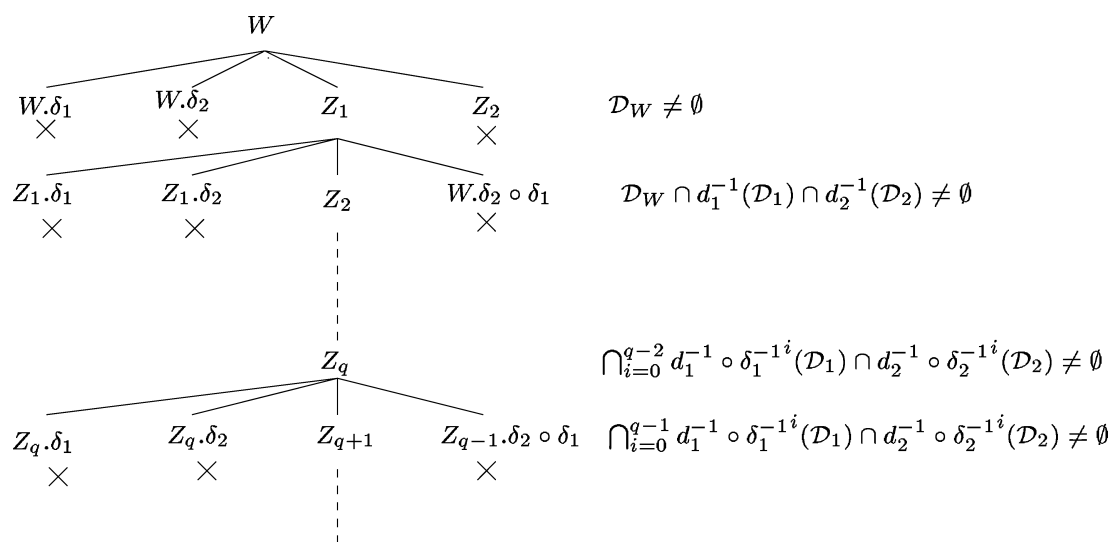

Fig. 12. Tree of added variables when there are multiple branches.
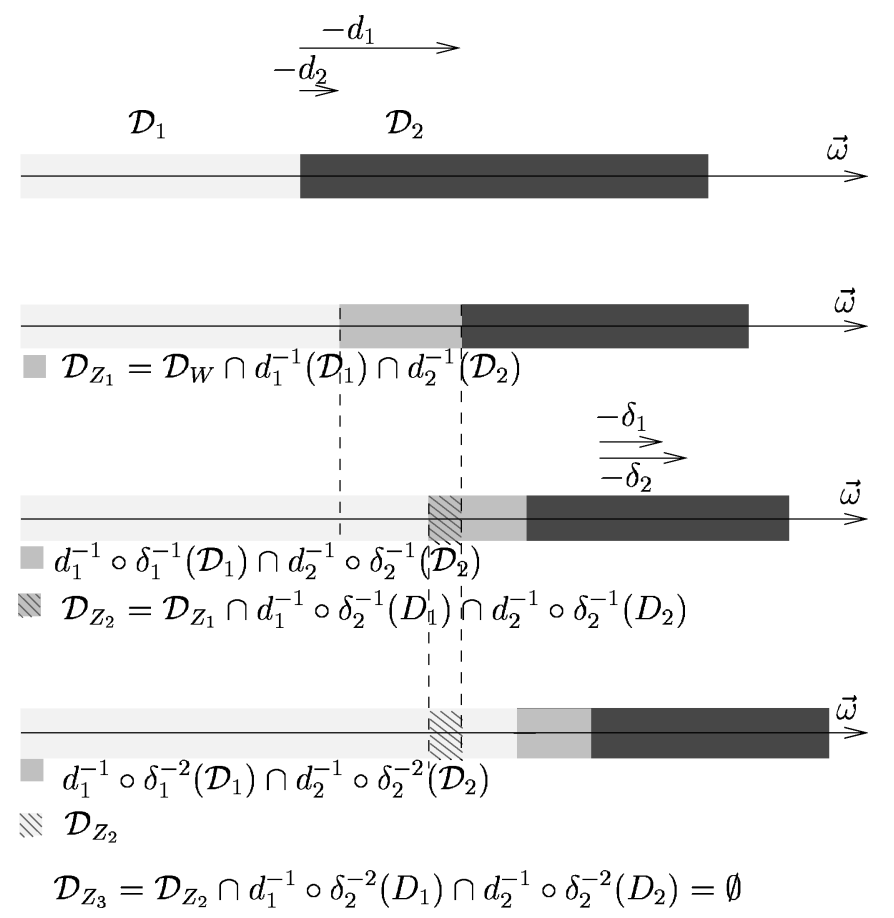

Fig. 13. Intersection of domains preimages, projections of $\delta_{1}$ and $\delta_{2}$ are negative.

of these projections. In addition, we compute the intersection with the domain of variable $Z_{q}$. These computations are illustrated on Figure 13 for an example where the projections of $\delta_{1}$ and $\delta_{2}$ along $\vec{\omega}$ are negative. For this example, we remark that

- the boundaries of intersection of preimages of $\mathcal{D}_{1}$ and $\mathcal{D}_{2}$ are respectively translated by $\delta_{1}$ and $\delta_{2}$,

- the interval resulting from the projection of this intersection along $\vec{\omega}$ strictly decreases in size at each iteration. 
In the general case, if we denote by $\alpha_{1}, \alpha_{2}, \beta_{1}, \beta_{2}$ the respective projections of $d_{1}, d_{2}, \delta_{1}, \delta_{2}$ along $\vec{\omega}$, this intersection becomes empty after a finite number of iterations if and only if $\alpha_{2}-\alpha_{1} \leq 0$ or $\beta_{2}-\beta_{1}<0$ or $\beta_{2}<0$ or $\beta_{1}>0$. The formal proof of this result is to be found in Appendix C. Intuitively, the negation of these conditions would mean that an instance of $X$ in a branch of its definition expression only depends on instances of $X$ in the same branch. In such a case, variable $X$ might be substituted by two variables not connected in the reduced dependence graph. We may assume without restriction that we have no such variables.

\subsection{Other Cases}

If the variable $X$ involved in the pattern variable $W$ depends on several distinct variables, the techniques described above applied in a systematic way would result in a combinatorial explosion. In order to limit the possible iteration space, we do not try to find an invariant in those branches where distinct variables occur.

Let us first consider $W$ defined by the following equation:

$$
W=\underset{i}{\vee} X \cdot d_{i} .
$$

- If $X$ is defined by

$$
X=\underset{i}{\vee} X_{i} \cdot d_{i}^{\prime}
$$

the iteration will consist of one single step, and thus be finite.

-If $X$ is defined by

$$
X=\stackrel{l}{\wedge} X_{i=1} \cdot d_{i}^{\prime}
$$

two cases may occur:

-if there is at most one occurrence of $X$ in $\left\{X_{1}, \ldots, X_{l}\right\}$, then the iteration will be finite;

-if there is strictly more than one occurrence of $X$ in $\left\{X_{1}, \ldots, X_{l}\right\}$, then the iteration will be infinite (this case resumes to the second case of Theorem 7.1).

-If $X$ is defined by

$$
X=\underset{j=1}{\vee} \stackrel{l_{j}}{\vee} X_{i=1} \cdot d_{i}^{\prime}
$$

the iteration will be finite if and only if each subterm $\wedge_{i=1}^{l_{j}} X_{i} . d_{i}^{\prime}$ corresponds to the first case above (at most one occurrence of $X$ ).

Note that in the conditions listed above, an occurrence of $\neg X$ is considered as a variable distinct from $X$.

Finally, if $W$ is defined by

$$
W=X . d_{1} \vee \neg X . d_{2}
$$

the iteration will stop after one single step. 


\section{IMPLEMENTATION}

The methods described below are implemented in MMALPHA, using the Polyhedral Library [Wilde 1993] for computations on polyhedra. Substitution and invariant searching are automatically performed, but the user still has to provide the exact domains on which these operations have to be made.

Each node represents a property. For each property we want to prove, we associate a proof tree. When building the proof tree, we simultaneously rewrite the system by performing substitutions: when, in a previously constructed node, a subexpression has been proved to have a given boolean value, we substitute all occurrences of this subexpression into the proof tree by this constant value. The proof tree is built as follows.

- The root node of the tree represents the toplevel property we want to prove.

- To construct the children of a node, we may apply five distinct rules:

- value-substitution: we substitute a variable by its value in the whole system.

-invariant and pattern detection: we first detect the pattern in the whole system, then two cases occur:

- either we can generate an invariant, thus we generate it and go on in building the proof tree,

- or the invariant generation is known to be infinite and we stop building the tree.

-negation rule: if the current goal is $\forall \rho, \rho \models_{\mathcal{D}} \neg e: v$, we generate a child node to prove $\forall \rho, \rho \models_{\mathcal{D}} e: \neg v$. The system remains unchanged.

- splitting rules: if the current goal is $\forall \rho, \rho \models_{\mathcal{D}} e_{1} \vee e_{2}:$ ff (respectively $\left.\forall \rho, \rho=_{\mathcal{D}} e_{1} \wedge e_{2}: t t\right)$, the problem is turned into proving that $\forall \rho,\left(\rho \models_{\mathcal{D}}\right.$ $e_{1}:$ ff $) \wedge\left(\begin{array}{lllll}\rho & \models_{\mathcal{D}} & e_{2} & : \text { ff }\end{array}\right)$ (respectively $\forall \rho,\left(\rho \models_{\mathcal{D}} e_{1}: t t\right) \wedge\left(\rho \models_{\mathcal{D}}\right.$ $\left.e_{2}: t t\right)$ ). We thus generate two children, one for each properties, and we add to the system two new variables defined by expressions $e_{1}$ and $e_{2}$.

-domain splitting rules: if the current goal is $\forall \rho, \rho=_{\mathcal{D}} e: v$, where $e$ is a case expression.

$$
e=\left\{\begin{array}{l}
\mathcal{D}_{1}: e_{1} \\
\vdots \\
\mathcal{D}_{n}: e_{n}
\end{array}\right.
$$

we generate $n$ children, one for each proof of $\forall \rho, \rho \models_{\mathcal{D}_{i}} e_{i}: v$.

When we apply one of these rules, we rewrite the system by performing substitutions, adding new variables and equations. This new system becomes the current system for the next nodes. These system transformations are propagated backwards in the proof tree to progress in the proof of previous siblings. We iterate the process until both system and proof tree remain unchanged.

-A node has no child if it represents one of the following cases:

- a constant expression

- an input variable

ACM Transactions on Embedded Computing Systems, Vol. 4, No. 2, May 2005. 
- an expression involving several distinct variables

- an expression that would lead to an infinite iteration.

- A node is valid if and only if all its children are valid.

The proof tree is built iteratively, and the building process is finite. To ensure this fact, we use a system of traces: when, in a tree branch, the same expression occurs twice, we stop the iteration. We know that we cannot generate infinitely new expressions, since the only way to get a new expression is to generate invariants, and we generate invariants only when we are sure that the generation is finite.

\section{APPLICATION TO THE MATRIX-VECTOR PRODUCT EXAMPLE: PERIODICITY AND VALIDITY}

Let us come back to the system computing a matrix product displayed in Figure 2. Recall that the problem is to show that we always have a significant result on the array output, i.e., for all $t$ in $\{t \mid N<t\}, c[t]$ is true. We are given an external specification of the system by means of the following statements.

$-b[t] \Longleftrightarrow \neg a[t-1] \wedge a[t]$,

$-b$ and $a$ are periodic signals,

- their common period is equal to $2 N$,

$-a$ is an alternation of $N$ true values and $N$ false values, the first true value being at $t=1$.

To prove that $c[t]$ is true for $t \in\{t, N \mid N<t\}$, we apply the substitution method: the problem is thus automatically turned into proving that $A[t, i]$ is true on domain $\{t, i, N \mid N<t ; i=N\}$. We cannot directly achieve the proof on this domain. We have to prove a more general result by widening this domain. If we simply widen it along the direction of dependency $t, i \rightarrow t-1, i-1$, we will have to prove that $A[t, i]$ is true on its whole domain of definition, which is obviously false (since input variable $a$ is not true everywhere). We thus have to choose a more precise widening. The only widening results in an infinite union of polyhedra (one for each period of $a$ ). As it is not directly possible in our model, we decompose our proof in two parts:

- we first prove that all our signals are periodic;

- we then restrict our study on a single period.

\subsection{Periodicity}

To prove the periodicity for all signals, we use the techniques we described in the previous sections: periodicity is just expressed by adding new equations in the system. Let $e[t]$ be a boolean periodic signal, of period $P$ : we have $e[t] \Leftrightarrow e[t-P]$. This equivalence is rewritten in conjunctive normal form. For each variable var representing one of the four local variables of our system, we automatically generate two variables defined by varper $1[i, t]=\operatorname{var}[t-P, i]$ or $\neg \operatorname{var}[t, i]$ and varper $2[i, t]=\operatorname{var}[t, i] \operatorname{or} \neg \operatorname{var}[t-P, i]$, and we prove that these two variables are true on their whole domain. 
We just sketch one of the eight proofs. We want to prove that variable Aper $1=$ $A[t-2 N, i]$ or not $A[t, i]$ is true on Domain $\{t, i \mid 0<=i<=N ; 2 N<=t\}$. Since dependencies are uniform, and since there is just one instance of $A$ in the definition of $A$, we are sure to find an invariant by using the invariant detection method. We are left with Aper 1 depending of Cper 1 , Aper 1, emptyper 1, A, not $A, C$, not $C$, empty, notempty. We repeat the process for every freshly introduced variable and we use the value-substitution method on every variable. All proofs automatically succeed.

\subsection{Considering One Period}

Since we work on periodic signals, the problem is now to prove that $A[t, i]$ is true on domain $\{t, i, N \mid N<t \leq 3 N ; i=N\}$. We use a heuristic procedure that takes the different dependencies into account to propose a way to widen the domain. The proof is restarted with this enlarged domain, yielding new proof obligations on other variables, which are handled the same way. There is one more case were the domain has to be widened (namely for variable $C$ ). At the end of the proof process, we get a precondition on input variables that must be initially ensured if we want $c$ to be true on the considered domain. In this particular example, the precondition we get is the following.

$$
b[1] \wedge \forall t \in\{t \mid 1 \leq t \leq N\}: a[t] .
$$

This precondition is a consequence of the external specification. ${ }^{6}$

\section{COMPLEXITY ISSUES}

The complexity of our overall method depends on three factors:

- the invariant detection heuristic is based on a search in a set of candidates; its complexity is exponential in the number of subterms of the patterns.

- operations on domains like computation of preimages and intersections are linear in the number of constraints defining these domains; in contrast, deciding if a domain is empty is based on Chernikova's algorithm [Le Verge 1992] and is exponential in the number of dimensions of the variables.

- the size of the proof tree is linear in the total number of subterms in the system, which in turn is exponential in the size of the system's expressions.

In practice, we manipulate systems with a few number of subterms. For instance, proving the periodicity of Init and Empty in Section 9 is only a matter of seconds. On the other hand, expressions with 16 subterms approximatively take 5 hours of computation on a standard workstation. The examples treated here involve at most 5 dimensions, but other tests (using additional proof methods not presented in this paper) show that our tool is able to handle up to 10 dimensions without running into complexity or numerical issues.

\footnotetext{
${ }^{6}$ In fact, the initial specification assumed $a[1]=b[0]=$ True and $b[1]=$ False, which lead to an error. Moreover, our proof method led to discover an error in the system itself.
} 
The overall complexity could be improved in two ways:

-instead of using the Polyhedral Library as a black box, we could select the functions we are interested in and adapt them to our particular context, by using less powerful but less complex algorithms.

-iterations in the construction of the proof tree are done in a naive way. We should definitely design cleverer algorithms to speed up this construction.

\section{RELATED WORK}

This work uses an approach based on substitutions earlier described in Cachera et al. [2001], where a similar method was used to prove functional equivalence of two SAREs. In this paper, we generalize this approach to safety properties on a given polyhedral domain, and give a new heuristic method to look for invariants when the simple approach fails.

Though many properties concerning parameterized SAREs are undecidable, recent works give heuristics for automatically checking equivalence of two SAREs in some particular cases. In Shashidar et al. [2002], an automatic tool for checking functional equivalence between two loop nests is described. It handles transformations limited to buffer copy and loop reorganization. In Barthou et al. [2001], the authors show the undecidability of the general case, and give a semialgorithm that is able to handle most of the usual SARE transformations (renaming, iteration space modifications, scheduling, and so on).

When dealing with parameterized systems, we might either use tools based on higher-order logics like theorem provers, or try to adapt methods initially devoted to finite-state systems. The former approach has been widely investigated in the general framework of formal hardware verification, combining various Hardware Description Languages with various theorem proving tools. In the polyhedral model framework, this approach has been investigated using two different proof assistants, namely PVS [Shankar et al. 2001] and Coq [The Coq Development Team, LogiCal Project]. In both approaches, SAREs are translated into the proof assistant specification language, and interactive proofs are partially automated through the use of specific strategies [Cachera et al. 2001; Cachera and Pichardie 2003]. A similar work has been done in Pierre [1995], where equivalence of VHDL descriptions (at bit level and at arithmetical level) is proved thanks to the Nqthm theorem prover. Generalization heuristics are used tor guess invariants for proving equivalence between recursive functions and iterative ones. This approach only applies to one- or two-dimensional architectures, and requires regularity to be expressed through precise and fixed constructs.

Even if Apt and Kozen [1986] have shown that model-checking techniques cannot be applied to regular networks of processes of unknown size, it is still possible to adapt this kind of techniques to parameterized systems in some particular cases. Many authors restrict themselves to the case of linear networks of processes. For instance, Halbwachs et al. [1992] prove mutual exclusion in a linear hardware arbiter. They rely on complex user-given invariants to prove the induction step from one parameter value to the next one. Such an approach is not easily generalizable. In Lesens et al. [2001], a more general approach to 
linear networks is developed, using observers and network invariants: invariants are expressed as fix-points, and over-approximations are found by widening techniques. In this kind of tool, the user has to adjust extrapolation parameters. Other approaches inspired from abstract interpretation try to automatically construct a finite approximation of an infinite-state system. In Saidi [2000], boolean abstraction is performed in the framework of guarded transition systems, using predicates over concrete variables as boolean abstract variables. Invariants are generated by static analysis techniques. The whole process consists in a series of refinements which, if successful, lead to a finite-state abstract description that can be model-checked. In contrast to these approaches, we do not try to define complex invariants to perform inductive proofs on the system size. We rely on the "natural" abstraction of the polyhedral model to handle parameters in a symbolic fashion, and our invariants are "temporal" invariants that focus only on the partial property we want to prove.

\section{CONCLUSION}

We have presented heuristic methods for proving control properties of regular parameterized systems described by means of affine recurrence equations. These methods take advantage of the computational power of the polyhedral model and of the way it naturally provides abstraction for generic systems. We combine syntactic substitutions that provide a simple induction principle, and invariant generation in a semiautomatic process.

When restricting ourselves to uniform dependencies and scheduled systems, we are able to identify cases where invariant searching will be useful. In practice, as we deal with low-level descriptions with few complex signal manipulations, these methods will prove successful in most cases. Even if our method aims at formally checking a safety property, it also can provide counterexamples in some cases of failure. A deeper interpretation of results in cases when the proof process has not completed would provide more bug-tracking features.

In this paper, we have illustrated our approach with simple examples extracted from real systems. In practice, control signals in existing systems are quite simple: they mainly involve initialisations and value propagations, or pipelined reductions over an array. Even if the inherent complexity of the proof steps is exponential in the number of expressions and dimensions, these numbers remain small enough to allow a proof construction in minutes. In that sense, our method is scalable to bigger benchmark examples, as long as control signals are not too deeply inter-dependent.

The properties we are able to check with the methods presented here are safety properties that do not involve existential quantification. More complex properties, like mutual exclusion for instance, are more difficult to express. They need to develop complementary proof techniques by artificially adding new parameters in the systems. Of course, the drawback of this approach is the increasing complexity. We are currently investigating this way to get more powerful but still usable proof techniques.

As we have seen in the matrix product example, it is often necessary to extend the polyhedral domain on which we try to prove a given property. In the 
current implementation, domain widening is performed thanks to very simple heuristics. We definitely should try to develop more specific widening operators.

We are currently investigating other ways to find invariants in those cases where the present techniques lead to an infinite iteration. The identification of specific patterns in polyhedral lattices seems to be a promising approach. We think that this could also provide a way to extend the class of properties we are able to prove, by considering not only safety properties, but also liveness properties.

\section{APPENDIX}

\section{A. PROOF OF PROPOSITION 5.3}

PRoposition 5.3. Let $\rho$ be a semantically correct environment, $X^{\prime}$ be defined as above, and $v$ a value. Then,

$$
\rho \models_{\mathcal{D}_{X}} X^{\prime}: v \Longleftrightarrow \rho \models_{\mathcal{D}_{X}} X: v .
$$

PRoof. The proof is made by strong induction on the temporal index $t_{z}$ of $z$, where $z$ is in $\mathcal{D}_{X}$. Let $t_{0}$ be a fixed value for the temporal index. Domain $D_{t_{0}}$ is a restriction of domain $D_{X}$ to those points whose value of temporal index is lower than $t_{0}$ :

$$
\mathcal{D}_{t_{0}}=\left\{z \mid t_{z} \leq t_{0} \wedge z \in \mathcal{D}_{X}\right\}
$$

First, we note that the sequence $\left(\mathcal{D}_{t}\right)_{t \in \mathbb{N}}$ of domains is increasing and converges to $\mathcal{D}_{X}$. Our induction hypothesis is the following.

$$
\forall z \in \mathcal{D}_{t_{0}}, \rho \models X^{\prime}[z]: v \Longleftrightarrow \forall z \in \mathcal{D}_{t_{0}}, \rho=X[z]: v .
$$

-For $t_{0}=0$, domain $D_{0}$ is a subset of $\mathcal{E} \mathcal{P}_{X}$. By definition of $X^{\prime}$, we know that on domain $\mathcal{E} \mathcal{P}_{X}, X^{\prime}=X$. Equivalence is trivial.

- We assume that our induction hypothesis is true for any value $t \leq t_{0}$, we must show that the following equivalence holds.

$$
\forall z \in \mathcal{D}_{t_{0}+1}, \rho \models X^{\prime}[z]: v \Longleftrightarrow \forall z \in \mathcal{D}_{t_{0}+1}, \rho \models X[z]: v .
$$

Let $z_{0}$ be a point of $\mathcal{D}_{t_{0}+1} \backslash \mathcal{D}_{t_{0}}$, then $z_{0}$ is either in $\left(\mathcal{D}_{t_{0}+1} \backslash \mathcal{D}_{t_{0}}\right) \cap \mathcal{E} \mathcal{P}_{X}$ or in $\mathcal{D}_{t_{0}+1} \backslash\left(\mathcal{D}_{t_{0}} \cup \mathcal{E} \mathcal{P}_{X}\right)$ :

-If $z_{0}$ is in $\left(\mathcal{D}_{t_{0}+1} \backslash \mathcal{D}_{t_{0}}\right) \cap \mathcal{E} \mathcal{P}_{X}$ : by definition of $X^{\prime}$ on this domain, we have $\rho\left(X^{\prime}\right)=\rho(X)$.

-If $z_{0}$ is in $\mathcal{D}_{t_{0}+1} \backslash\left(\mathcal{D}_{t_{0}} \cup \mathcal{E} \mathcal{P}_{X}\right)$ : on this domain, we know that $X$ is defined by the following equation:

$$
X=g\left(\ldots, X . d_{i}, \ldots\right) .
$$

As we work on a scheduled system, $d_{i}\left(z_{0}\right)$ is in $\mathcal{D}_{t_{0}}$. Let us now assume that $\forall z \in \mathcal{D}_{t_{0}+1}, \rho \models X^{\prime}[z]: v$, in particular, we have $\forall z \in \mathcal{D}_{t_{0}}, \rho \models X^{\prime}[z]: v$. By our induction hypothesis, we know that $\forall z \in \mathcal{D}_{t_{0}}, \rho \models X[z]: v$. This in particular applies to $d_{i}\left(z_{0}\right)$. As a consequence, for any $z$ in $\mathcal{D}_{t_{0}+1} \backslash\left(\mathcal{D}_{t_{0}} \cup \mathcal{E} \mathcal{P}_{X}\right)$, we have $\rho \models X\left[d_{i}(z)\right]: v$. Due to Lemma (5.2) on substitutions, expression $g\left(\ldots, X . d_{i}, \ldots\right)$ is semantically equivalent to $g(\ldots, v, \ldots)$. Since $\rho$ is semantically correct, and by definition of $X^{\prime}$, we can conclude that

$$
\begin{aligned}
& \forall z \in \mathcal{D}_{t_{0}+1} \backslash\left(\mathcal{D}_{t_{0}} \cup \mathcal{E} \mathcal{P}_{X}\right), \rho \models X^{\prime}[z]: v \Longrightarrow \\
& \forall z \in \mathcal{D}_{t_{0}+1} \backslash\left(\mathcal{D}_{t_{0}} \cup \mathcal{E} \mathcal{P}_{X}\right), \rho \models X[z]: v .
\end{aligned}
$$


Conversely, if we assume that $\forall z$ in $\mathcal{D}_{t_{0}+1}, \rho \models X[z]: v$, we prove in the same way that

$$
\begin{aligned}
& \forall z \in \mathcal{D}_{t_{0}+1} \backslash\left(\mathcal{D}_{t_{0}} \cup \mathcal{E} \mathcal{P}_{X}\right), \rho \models X^{\prime}[z]: v \\
& \Longleftarrow \forall z \in \mathcal{D}_{t_{0}+1} \backslash\left(\mathcal{D}_{t_{0}} \cup \mathcal{E} \mathcal{P}_{X}\right), \rho \models X[z]: v .
\end{aligned}
$$

\section{B. CASE OF A SINGLE BRANCH: FORMAL PROOF FOR $X=\wedge_{i} X . d_{i}^{\prime}$}

LEMma B.1. Let $d_{1}, d_{2}, \delta_{1}, \delta_{2}$ be uniform dependencies. Let $n$ be a positive integer. Let $S_{1}^{n}, S_{2}^{n}, S_{3}^{n}, S_{4}^{n}$ be the four systems defined by the following equations.

$$
\begin{aligned}
& S_{1}^{n}=\left\{\begin{array}{l}
\delta_{1}^{n} \circ d_{1}=d_{1} \circ f \\
\delta_{2}^{n} \circ d_{2}=d_{2} \circ f \\
\delta_{2} \circ d_{1}=d_{1} \circ f
\end{array} \quad S_{3}^{n}=\left\{\begin{array}{l}
\delta_{1}^{n} \circ d_{1}=d_{2} \circ f \\
\delta_{2}^{n} \circ d_{2}=d_{1} \circ f \\
\delta_{2} \circ d_{1}=d_{2} \circ f
\end{array}\right.\right. \\
& S_{2}^{n}=\left\{\begin{array}{l}
\delta_{2}^{n} \circ d_{1}=d_{1} \circ f \\
\delta_{1}^{n} \circ d_{2}=d_{2} \circ f
\end{array} \quad S_{4}^{n}=\left\{\begin{array}{l}
\delta_{2}^{n} \circ d_{1}=d_{2} \circ f \\
\delta_{1}^{n} \circ d_{2}=d_{1} \circ f .
\end{array}\right.\right.
\end{aligned}
$$

Systems $S_{1}^{n}$, $S_{2}^{n}$ have no solution. If system $S_{3}^{n}$ has a solution, system $S_{4}^{n}$ does not and conversely.

PRoof. Since $\delta_{1}$ and $\delta_{2}$ are not equal, systems $S_{1}^{n}$ and $S_{2}^{n}$ have no solution. We now prove the last assertion. Let us suppose that $S_{3}^{n}$ has a solution, then we have

$$
\delta_{1}^{n} \circ d_{1}^{2}=\delta_{2}^{n} \circ d_{2}^{2} .
$$

Similarly, if $S_{4}^{n}$ has a solution, we have

$$
\delta_{1}^{n} \circ d_{2}^{2}=\delta_{2}^{n} \circ d_{3}^{2} .
$$

By combining equations (13) and (14), we get $d_{1}^{4}=d_{2}^{4}$. Since $d_{1}$ and $d_{2}$ are uniform dependencies, $d_{1}^{4}=d_{2}^{4}$ is equivalent to $d_{1}=d_{4}$. As a consequence, systems $S_{3}^{n}$ and $S_{4}^{n}$ cannot simultaneously have a solution.

\section{FORMAL PROOF OF THE CASE OF MULTIPLE BRANCHES}

To decide the existence of such a $q$, we embed the problem into $\mathbb{R}^{p}$. Let us recall that $\mathcal{D}_{1}$ is the domain of the first branch of $X$ and $\mathcal{D}_{2}$ the domain of the second branch. By definition of domains, we know that they are delimited by a finite number of hyperplanes and that their intersection is empty, so there exists an hyperplane $P$, and two half-spaces $P_{1}$ and $P_{2}$ defined such that $\mathcal{D}_{1} \subset P_{1}$ and $\mathcal{D}_{2} \subset P_{2}, P_{1}$ and $P_{2}$ will be defined as follows. Let $P$ be an hyperplane of dimension $p-1$, and $\vec{\omega}$ be a vector orthogonal to this hyperplane. Hyperplane $P$ is generated by a free family of vectors $v_{1}, \ldots, v_{p-1}$. Family $v_{1}, \ldots, v_{p-1}, \vec{\omega}$ is a basis of $\mathbb{R}^{p}$. Let us denote by $P_{1}$ and $P_{2}$ the open half-spaces delimited by $P$ such that $P_{1}=\{x \mid x=y+\alpha \vec{\omega}, \alpha<0, y \in P\}$ and $P_{2}=\{x \mid x=y+\alpha \vec{\omega}, \alpha>$ $0, y \in P\}$. Let us now consider the uniform dependencies $d_{1}, d_{2}, \delta_{1}, \delta_{2}$ defined for every index vector $x$.

$$
\begin{aligned}
d_{1}(x) & =x+\sum_{i=1}^{p-1} \alpha_{1, i} v_{i}+\alpha_{1} \vec{\omega} \\
d_{2}(x) & =x+\sum_{i=1}^{p-1} \alpha_{2, i} v_{i}+\alpha_{2} \vec{\omega} \\
\delta_{1}(x) & =x+\sum_{i=1}^{p-1} \beta_{1, i} v_{i}+\beta_{1} \vec{\omega} \\
\delta_{2}(x) & =x+\sum_{i=1}^{p-1} \beta_{2, i} v_{i}+\beta_{2} \vec{\omega} .
\end{aligned}
$$

ACM Transactions on Embedded Computing Systems, Vol. 4, No. 2, May 2005. 
Since we work in $\mathbb{R}^{p}$, our problem is turned into proving the existence of a $q$ such that,

$$
\bigcap_{i=0}^{q} d_{1}^{-1} \circ\left(\delta_{1}^{-1}\right)^{i}\left(P_{1}\right) \cap d_{2}^{-1} \circ\left(\delta_{2}^{-1}\right)^{i}\left(P_{2}\right)=\emptyset .
$$

Since all the dependencies are translations, and since we work on half-spaces, the image of one of the half-spaces (e.g. $\left.P_{1}\right)$ is a translation of the half-space $\left(P_{1}\right)$ along vector $\vec{\omega}$. The vacuity of the intersection of images of $P_{1}$ and $P_{2}$ by these dependencies only depends on the coefficients of the projection of dependencies $d_{1}, d_{2}, \delta_{1}, \delta_{2}$ on vector $\vec{\omega}$. We denote by $\alpha_{1}, \alpha_{2}, \beta_{1}$, and $\beta_{2}$ there respective projections.

In the following, we will give necessary and sufficient conditions so that the intersection of (15) is empty in $\mathbb{R}^{p}$.

Theorem C.1. Let $W$ and $X$ be two polyhedral variables defined by the following equations.

$$
\begin{aligned}
& W=\mathcal{D}_{W}: X . d_{1} \vee X . d_{2} \\
& X=\left\{\begin{array}{l}
\mathcal{D}_{1}: X . \delta_{1} \\
\mathcal{D}_{2}: X . \delta_{2} .
\end{array}\right.
\end{aligned}
$$

Iteration of invariant generation and value substitution is finite if and only if $\alpha_{2}-\alpha_{1} \leq 0$ or $\beta_{2}-\beta_{1}<0$ or $\beta_{2}<0$ or $\beta_{1}>0$.

These conditions intuitively mean that there is an "interaction" between branches of $X$, that is, that there exist instances of $X$ in some branches that depend on instances in other branches. The negation of these conditions would mean that an instance of $X$ in a branch of its definition expression only depends on instances of $X$ in the same branch. In such a case, variable $X$ might be substituted by two variables not connected in the reduced dependence graph. We may assume without restriction that we have no such variables.

We decompose the proof of Theorem C.1 into several propositions. For the first, we embed the problem into $\mathbb{R}^{p}$.

Proposition C.2.

$$
\begin{aligned}
& \alpha_{2}-\alpha_{1}>0 \wedge \beta_{2}-\beta_{1} \geq 0 \wedge \beta_{1} \leq 0 \wedge \beta_{2} \geq 0 \\
\Longleftrightarrow & \forall q \in \mathbb{N}, \bigcap_{i=1}^{q} d_{1}^{-1} \circ\left(\delta_{1}^{-1}\right)^{i}\left(P_{1}\right) \cap d_{2}^{-1} \circ\left(\delta_{2}^{-1}\right)^{i}\left(P_{2}\right) \neq \emptyset .
\end{aligned}
$$

Proof. Let $A_{i}$ be $d_{1}^{-1} \circ\left(\delta_{1}^{-1}\right)^{i}\left(P_{1}\right) \cap d_{2}^{-1} \circ\left(\delta_{2}^{-1}\right)^{i}\left(P_{2}\right)$.

$$
\forall q \in \mathbb{N}, \bigcap_{i=1}^{q} A_{i} \neq \emptyset
$$

is equivalent to

$$
\forall q \in \mathbb{N}, \exists x, \forall 0 \leq j, j^{\prime} \leq q, x \in A_{j} \cap A_{j^{\prime}} .
$$

Let $x$ be a point such that

$$
\forall 0 \leq j, j^{\prime} \leq q, x \in A_{j} \cap A_{j^{\prime}}
$$

Thus, there exist $y_{1, j}, y_{1, j^{\prime}}^{\prime}$ in $P_{1}$ such that

$$
x=d_{1}^{-1} \circ\left(\delta_{1}^{-1}\right)^{j}\left(y_{1, j}\right)=d_{1}^{-1} \circ\left(\delta_{1}^{-1}\right)^{j^{\prime}}\left(y_{1, j^{\prime}}^{\prime}\right)
$$


and $y_{2, j}, y_{2, j^{\prime}}^{\prime}$ in $P_{2}$ such that

$$
x=d_{2}^{-1} \circ\left(\delta_{2}^{-1}\right)^{j}\left(y_{2, j}\right)=d_{2}^{-1} \circ\left(\delta_{2}^{-1}\right)^{j^{\prime}}\left(y_{2, j^{\prime}}^{\prime}\right) .
$$

Let us denote by $x_{\vec{\omega}}$ the projection of $x$ along vector $\vec{\omega}$. Since $y_{1, j}, y_{1, j^{\prime}}^{\prime}$ are in $P_{1}$ and $y_{2, j}, y_{2, j^{\prime}}^{\prime}$ in $P_{2}$, there exist $\gamma_{1, j}, \gamma_{1, j^{\prime}}^{\prime}>0$ and $\gamma_{2, j}, \gamma_{2, j^{\prime}}^{\prime}<0$, where $\gamma_{1, j}$ (respectively $\gamma_{1, j^{\prime}}^{\prime}, \gamma_{2, j}, \gamma_{2, j^{\prime}}^{\prime}$ ) is the projection along vector $\vec{\omega}$ of $y_{1, j}$ (respectively $\left.y_{1, j^{\prime}}, y_{2, j}, y_{2, j^{\prime}}^{\prime}\right)$ such that

$$
\begin{gathered}
x_{\vec{\omega}}=\left(\gamma_{1, j}-\alpha_{1}-j \beta_{1}\right) \vec{\omega}=\left(\gamma_{2, j}-\alpha_{2}-j \beta_{2}\right) \vec{\omega} \\
=\left(\gamma_{1, j^{\prime}}^{\prime}-\alpha_{1}-j^{\prime} \beta_{1}\right) \vec{\omega}=\left(\gamma_{2, j^{\prime}}^{\prime}-\alpha_{2}-j^{\prime} \beta_{2}\right) \vec{\omega} .
\end{gathered}
$$

Using equation (17), expression (16) may thus be formulated as follows,

$$
\begin{gathered}
\forall q \in \mathbb{N}, \quad \forall 0 \leq j, j^{\prime} \leq q \exists \gamma_{1, j}, \gamma_{1, j^{\prime}}^{\prime}>0 \gamma_{2, j}, \gamma_{2, j^{\prime}}^{\prime}<0 \\
\gamma_{1, j}-\alpha_{1}-j \beta_{1}=\gamma_{2, j}-\alpha_{2}-j \beta_{2} \\
\gamma_{1, j}-\alpha_{1}-j \beta_{1}=\gamma_{2, j^{\prime}}^{\prime}-\alpha_{2}-j^{\prime} \beta_{2} \\
\gamma_{1, j^{\prime}}^{\prime}-\alpha_{1}-j^{\prime} \beta_{1}=\gamma_{2, j}-\alpha_{2}-j \beta_{2} .
\end{gathered}
$$

Since equality (18) is true for all $0 \leq j \leq q$, it is true for $j=0$. It follows that $\gamma_{2,0}-\gamma_{1,0}=\alpha_{2}-\alpha_{1}$. Hence, we have $\exists \gamma_{1,0}<0, \gamma_{2,0}>0, \gamma_{2,0}-\gamma_{1,0}=\alpha_{2}-\alpha_{1}$ which is equivalent to $\alpha_{2}>\alpha_{1}$

Since $\gamma_{2, j^{\prime}}^{\prime}>0$ and $\gamma_{1, j}<0$, we can transform equality (19) into the following inequality

$$
\alpha_{2}-\alpha_{1}+j^{\prime} \beta_{2}-j \beta_{1}>0 .
$$

If $j^{\prime}=0$ and $j=q$ we have

$$
\alpha_{2}-\alpha_{1}>q \beta_{1} .
$$

So $\frac{\alpha_{2}-\alpha_{1}}{q} \geq \beta_{1}$. As this inequality must be true for any value of $q \in \mathbb{N}$, this implies that $\beta_{1} \leq 0$. Similarly, using equation (20), we obtain $\beta_{2} \geq 0$. Conversely, if $\alpha_{2}-\alpha_{1}>0 \wedge \beta_{2}-\beta_{1} \geq 0 \wedge \beta_{1} \leq 0 \wedge \beta_{2} \geq 0$, there exist $\gamma_{1, j}, \gamma_{1, j^{\prime}}^{\prime}<0, \gamma_{2, j}, \gamma_{2, j^{\prime}}^{\prime}>0$, such that equalities (18), (19), and (20) hold.

We can now apply Proposition (C.2) to polyhedra.

Proposition C.3. If $\alpha_{2}-\alpha_{1} \leq 0$ or $\beta_{2}-\beta_{1}<0$ or $\beta_{2}<0$ or $\beta_{1}>0$, there exists $q$ such that

$$
\bigcap_{i=1}^{q} d_{1}^{-1} \circ\left(\delta_{1}^{-1}\right)^{i}\left(\mathcal{D}_{1}\right) \cap d_{2}^{-1} \circ\left(\delta_{2}^{-1}\right)^{i}\left(\mathcal{D}_{2}\right)=\emptyset .
$$

Remark that if $q=0$ the equivalence can be rewritten into

$$
\alpha_{2}-\alpha_{1}>0 \Longleftrightarrow d_{1}^{-1}\left(D_{1}\right) \cap d_{2}^{-1}\left(D_{2}\right) \neq \emptyset .
$$

This remark leads us to give Corollary (C.4). This corollary allows us to study only one branch of the two last branches defining variable $W$, since the domain of the other one will be empty. 
Corollary C.4.

$$
\begin{aligned}
& d_{1}^{-1}\left(\mathcal{D}_{1}\right) \cap d_{2}^{-1}\left(\mathcal{D}_{2}\right) \neq \emptyset \Longrightarrow d_{1}^{-1}\left(\mathcal{D}_{2}\right) \cap d_{2}^{-1}\left(\mathcal{D}_{1}\right)=\emptyset \\
& d_{1}^{-1}\left(\mathcal{D}_{2}\right) \cap d_{2}^{-1}\left(\mathcal{D}_{1}\right) \neq \emptyset \Longrightarrow d_{1}^{-1}\left(\mathcal{D}_{1}\right) \cap d_{2}^{-1}\left(\mathcal{D}_{2}\right)=\emptyset
\end{aligned}
$$

\section{ACKNOWLEDGMENTS}

The authors would like to thank Patrice Quinton for fruitful discussions on this topic.

\section{REFERENCES}

Alur, R., Grosu, R., Hur, Y., Kumar, V., And Lee, I. 2000. Modular specification of hybrid systems in CHARON. In Proceedings of the 3rd International Workshop on Hybrid Systems: Computation and Control, N. Lynch and B. Krogh, Eds. Lecture Notes in Computer Science, vol. 1790. Pittsburgh, PA, 6-19.

APT, K. AND KozEN, D. 1986. Limits for automatic verification of finite-state concurrent systems. Information Processing Letters 22, 307-309.

Asarin, E., Dang, T., and Maler, O. 2002. The d/dt tool for verification of hybrid systems. In Lecture Notes in Computer Science, vol. 2404, 365-369.

Barthou, D., Feautrier, P., and Redon, X. 2001. On the Equivalence of Two Systems of Affine Recurrence Equations. Tech. Rep. 4285, INRIA.

CACHera, D. And Pichardie, D. 2003. Embedding of systems of affine recurrence equations in Coq. In Proceedings of Theorem Proving in Higher Order Logics 2003, Roma LNCS, vol. 2758.

Cachera, D., Quinton, P., Rajopadhye, S., and Risset, T. 2001. Proving properties of multidimensional recurrences with application to regular parallel algorithms. In FMPPTA'01, San Francisco, CA.

Derrien, S., Guillou, A., Quinton, P., Risset, T., And Wagner, C. 2002. Automatic synthesis of efficient interfaces for compiled regular architectures. In Workshop on Systems, Architectures, Modeling, and Simulation (SAMOS).

Guillou, A., Quinton, P., Risset, T., And Massicotte, D. 2000. Automatic design of VLSI pipelined LMS architectures. In Parelec'2000.

Halbwachs, N., Lagnier, F., AND Ratel, C. 1992. An experience in proving regular networks of processes by modular model checking. Acta Informatica 29, 523-543.

Henzinger, T. A., Preussig, J., And Wong-Toi, H. 2001. Some lessons from the HyTech experience. In Proceedings of 40th Annual Conference on Decision and Control. IEEE Computer Society Press, Los Alamitos, CA. 2887-2892.

Karp, R., Miller, R., And Winograd, S. 1967. The organization of computations for uniform recurrence equations. Journal of the Association for Computing Machinery 14, 3 (July), 563-590.

Le Verge, H. 1992. A Note on Chernikova's Algorithm. Tech. Rep. 635, IRISA.

Lesens, D., Halbwachs, N., And Raymond, P. 2001. Automatic verification of parameterized linear networks of processes. Theoretical Computer Science 255.

Manjunathaiah, M., Megson, G., Rajopadhye, S., and Risset, T. $2001 . \quad$ Uniformization tool for systolic array designs. In ICPP 01.

MaURAS, C. 1989. Alpha : un langage équationnel pour la conception et la programmation d'architectures systoliques. Ph.D. Thesis, Univ. Rennes I, France.

PIERRE, L. 1995. An automatic generalization method for the inductive proof of replicated and parallel architectures. In Theorem Provers in Circuit Design. LNCS, vol. 901.

Quinton, P., Rajopadhye, S., And Risset, T. 1996. On Manipulating Z-Polyhedra. Tech. Rep. 1016, IRISA, Rennes, France. Jan.

SAIDI, H. 2000. Model checking guided abstraction and analysis. In The 7th International Static Analysis Symposium.

SAouter, Y. AND Quinton, P. 1993 Computability of recurrence equations. Theoretical Computer Science 116.

SchriJver, A. 1986. Theory of Linear and Integer Programming. Wiley, New York. 
Shankar, N., Owre, S., Rushby, J. M., and Stringer-Calvert, D. W. J. 2001. PVS Prover Guide. Computer Science Laboratory, SRI International, Menlo Park, CA.

Shashidar, K. C., Catthoor, M. B. F., And Janssens, H. 2002. Geometric model checking: An automatic verification technique for loop and data reuse transformations. In International Workshop on Compiler Optimization Meets Compiler Verification (COCV). 71-86.

Silva, B. I. AND KROGH, B. H. 2000. Formal verification of hybrid system using CheckMate: A case study. In American Control Conference.

The Coq Development Team, LogiCal Project. The Coq proof Assistant, Reference Manual.

WiLDE, D. 1993. A Library for Doing Polyhedral Operations. Tech. Rep. 785, IRISA, Rennes, France.

WiLDE, D. 1994. The Alpha Language. Tech. Rep. 999, IRISA, Rennes, France.

Received September 2003; revised February 2004; accepted June 2004 